\title{
SOME HIIBERT SPACES OF ENTIRE FUNCTIONS. II
}

\author{
BY \\ LOUIS DE BRANGES(1)
}

A Hilbert space, whose elements are entire functions, is of especial interest if it has these three properties:

(H1). Whenever $F(z)$ is in the Hilbert space and has a nonreal zero $w$, $F(z)(z-\bar{w}) /(z-w)$ is in the Hilbert space and has the same norm as $F(z)$.

(H2). Whenever $w$ is a nonreal complex number, the linear functional defined on the Hilbert space by $F(z) \rightarrow F(w)$ is continuous.

(H3). Whenever $F(z)$ is in the Hilbert space, the function $F^{*}(z)=\bar{F}(\bar{z})$ is in the Hilbert space and has the same norm as $F(z)$.

Let $E(z)$ be an entire function such that

$$
|E(\bar{z})|<|E(z)|
$$

for $y>0(z=x+i y)$. We write $E(z)=A(z)-i B(z)$ where $A(z)$ and $B(z)$ are entire functions which are real for real $z$ and

$$
K(w, z)=[B(z) \bar{A}(w)-A(z) \bar{B}(w)] /[\pi(z-\bar{w})] .
$$

Then, the set $\mathfrak{F}(E)$ of entire functions $F(z)$ such that

$$
\|F\|^{2}=\int|F(t)|^{2}|E(t)|^{-2} d t<\infty
$$

with integration on the real axis, and

$$
|F(z)|^{2} \leqq\|F\|^{2} K(z, z)
$$

for all complex $z$, is a Hilbert space of entire functions satisfying (H1), (H2), and $(\mathrm{H} 3)$. For each complex number $w, K(w, z)$ belongs to $\Im(E)$ as a function of $z$ and

$$
F(w)=\langle F(t), K(w, t)\rangle .
$$

By [7], every Hilbert space of entire functions which satisfies (H1), (H2), $(\mathrm{H} 3)$, and which contains a nonzero element, is equal isometrically to some such $\operatorname{He}(E)$.

Computations involving these Hilbert spaces use matrix valued entire functions

Received by the editors August 16, 1960.

(1) This research was supported by the United States Air Force through the Air Force Office of Scientific Research of the Air Research and Development Command, under contract No. AF49(638)-253. 


$$
M(z)=\left(\begin{array}{ll}
A(z) & B(z) \\
C(z) & D(z)
\end{array}\right),
$$

where $A(z), B(z), C(z), D(z)$ are real for real $z$ and

$$
\begin{aligned}
A(z) D(z)-B(z) C(z) & =1, \\
\operatorname{Re}[A(z) \bar{D}(z)-B(z) \bar{C}(z)] & \geqq 1, \\
{[B(z) \bar{A}(z)-A(z) \bar{B}(z)] /(z-\bar{z}) } & \geqq 0, \\
{[D(z) \bar{C}(z)-C(z) \bar{D}(z)] /(z-\bar{z}) } & \geqq 0,
\end{aligned}
$$

for all complex $z$. If $u$ and $v$ are complex numbers such that

$$
|u-i v|=|u+i v|
$$

and if $u$ and $v$ are not both zero, we use the condition

$$
[D(i y) v+i C(i y) v+i A(i y) u+B(i y) u]
$$

as $y \rightarrow+\infty$.

$$
=o(y[A(i y) v-i B(i y) v+i D(i y) u-C(i y) u])
$$

TheOREM I. Let $E(z)$ be an entire function satisfying (1). A necessary and sufficient condition that the elements $F(z)$ of $\operatorname{HC}(E)$ whose product by z belongs to $\mathrm{JC}(E)$ fail to be dense in $\mathrm{JC}(E)$ is that there be complex numbers $u$ and $v$, not both zero, such that

$$
G(z)=A(z) u+B(z) v
$$

belongs to $\operatorname{HC}(E)$. In this case, they satisfy (3) and $G(z)$ spans the orthogonal complement in $\mathrm{F}(E)$ of such elements $F(z)$. The orthogonal complement of $G(z)$ in $\mathfrak{H}(E)$ satisfies (H1), (H2), and (H3).

TheOREM II. Let $E(a, z)$ be an entire function satisfying (1). Let $M(a, b, z)$ be a matrix valued entire function satisfying (2). If there exist complex numbers $u$ and $v$ such that $G(z)$, defined by (5) for $E(z)=E(a, z)$, belongs to $\operatorname{xc}(E(a))$, we suppose that $M(a, b, z)$ satisfies (4) for this choice of $u$ and $v$. Let

$$
E(b, z)=A(b, z)-i B(b, z)
$$

where

$$
(A(b, z), B(b, z))=(A(a, z), B(a, z)) M(a, b, z) .
$$

Then, $E(b, z)$ satisfies (1) and $\mathfrak{H C}(E(a))$ is contained isometrically in $\operatorname{HC}(E(b))$. For each real number $w$, there exists an element $F(z)$ of $\mathcal{J C}(E(a))$ such that

$$
F(w) / E(b, w) \neq 0 \text {. }
$$

THEOREM III. Let $E(a, z)$ and $E(z)$ be entire functions satisfying (1) and such that $\operatorname{HC}(E(a))$ is contained isometrically in $\operatorname{HC}(E)$. Suppose that for each real number $w$, there is an element $F(z)$ of $\operatorname{He}(E(a))$ such that $F(w) / E(w) \neq 0$. 
Then, $E(z)=E(b, z)$ for some choice of $M(a, b, z)$ as in Theorem II. In this case, $M(a, b, z)$ is uniquely determined by $E(a, z)$ and $E(z)$.

Our purpose is to investigate the ideas associated with Theorem $\mathrm{X}$ of [8], which grew out of Sturm-Liouville differential equations. Let

$$
m(t)=\left(\begin{array}{ll}
\alpha(t) & \beta(t) \\
\beta(t) & \gamma(t)
\end{array}\right)
$$

be a matrix valued function of $t>0$ where $\alpha(t), \beta(t), \gamma(t)$ are real valued, absolutely continuous functions of $t>0$ such that

$$
\alpha^{\prime}(t) \geqq 0, \quad \gamma^{\prime}(t) \geqq 0, \quad \beta^{\prime}(t)^{2} \leqq \alpha^{\prime}(t) \gamma^{\prime}(t),
$$

a.e., for $t>0$. We also suppose that

$$
\alpha(0)=\lim _{t \supset 0} \alpha(t), \quad \int_{0}^{1}[\alpha(t)-\alpha(0)] d \gamma(t)
$$

exist and are finite. An interesting special case occurs when

$$
\beta(0)=\lim _{t \succ 0} \beta(t), \quad \gamma(0)=\lim _{t \searrow 0} \gamma(t)
$$

exist and are finite. At times, we also use the condition

$$
\lim _{t \rightarrow \infty}[\alpha(t)+\gamma(t)]=\infty
$$

A real number $b>0$ is said to be singular with respect to $m(t)$ if it belongs to an open interval $(a, c)$ in which $\alpha^{\prime}(t), \beta^{\prime}(t), \gamma^{\prime}(t)$ are equal a.e. to constant multiples of a single function and $\beta^{\prime}(t)^{2}=\alpha^{\prime}(t) \gamma^{\prime}(t)$. Otherwise, $t$ is said to be regular with respect to $m(t)$. Let

$$
I=\left(\begin{array}{rr}
0 & -1 \\
1 & 0
\end{array}\right)
$$

THEOREM IV. Let $m(t)$ be a matrix valued function of $t>0$ satisfying (7) and (8) and such that $\alpha(t)>\alpha(0)$ for $t>0$. Then, there exists a unique family $(E(t, z))$ of entire functions satisfying $(1), t \geqq 0$, such that for each complex number $w, E(t, w)$ is a continuous function of $t>0$, and

$$
(A(b, w), B(b, w)) I-(A(a, w), B(a, w)) I=w \int_{a}^{b}(A(t, w), B(t, w)) d m(t)
$$

whenever $a<b$ and

$$
\lim E(t, w) \exp [\beta(t) w]=1
$$

as $t \rightarrow 0$. For each fixed $a>0, E(a, z)$ has genus 0 or 1 and has no real zeros. If $a<b$ are regular points with respect to $m(t)$, then $\operatorname{HC}(E(a))$ is contained iso- 
metrically in $\operatorname{HC}(E(b))$. If the interval $(a, b)$ contains only singular points, the orthogonal complement of $\mathfrak{F C}(E(a))$ in $\mathfrak{H C}(E(b))$ is one-dimensional, unless $m(t)$ is a constant in $[a, b]$, in which case $E(a, z)=E(b, z)$.

THEOREM V. Let $E(z)$ be an entire function of genus 0 or 1 which satisfies (1). Then,

$$
E(z)=G(z) E(c, z)
$$

for some $c>0$, where $E(t, z)$ is defined as in Theorem IV for some choice of $m(t)$, and $G(z)$ is an entire function of genus 0 or 1 with only real zeros, such that

$$
G(z) G^{*}(w)=G^{*}(z) G(w)
$$

for all complex $z$ and $w$. If $E(z)$ has no real zeros and if $E(0)=1, m(t)$ may be chosen so that $G(z)=1$ identically. If $E^{*}(z)=E(-z)$ for all complex $z, m(t)$ may be chosen so that $\beta(t)=0$ identically.

If $m(t)$ satisfies (9) or (10), the construction has further properties.

THEOREM VI. Let $m(t)$ be a matrix valued function of $t>0$ satisfying (7), (8), and (9). Then, for each complex number w, there exists a unique continuous matrix valued function $M(t, w)$ of $t \geqq 0$ such that

$$
M(a, w) I-I=w \int_{0}^{a} M(t, w) d m(t),
$$

for $a \geqq 0$. For each fixed $a \geqq 0, M(a, z)$ is a matrix valued entire function of $z$ satisfying (2).

THEOREM VII. If $M(z)$ is a matrix valued entire function of $z$ satisfying (2) and such that $M(0)=1$, then

$$
M(z)=M(a, z)
$$

for some $a \geqq 0$, where $M(t, z)$ is defined as in Thorem $v 1$ jor some choice of $m(t)$.

TheOREM VIII. If, in the situation of Theorem IV, $m(t)$ satisfies (10), then

$$
K(t, z, z) \rightarrow \infty
$$

as $t \rightarrow \infty$, for all nonreal complex $z$. There is a unique nonnegative measure $\mu$ on the Borel sets of the real line such that for each $a>0$,

$$
\frac{y}{\pi} \int \frac{|E(a, t)|^{2} d \mu(t)}{(t-x)^{2}+y^{2}}=\lim _{b \rightarrow \infty} \frac{y}{\pi} \int \frac{|E(a, t)|^{2}|E(b, t)|^{-2} d t}{(t-x)^{2}+y^{2}},
$$

for $y>0$, and $\operatorname{se}(E(a))$ is contained isometrically in $L^{2}(\mu)$ whenever a is regular with respect to $m(t)$. The union of such $\operatorname{HC}(E(a))$, with a regular, is dense in $L^{2}(\mu)$.

For estimates of size, think of the $2 \times 2$ matrices with complex entries as 
determining linear transformations in complex euclidean 2-space, thought of as a Hilbert space, and use the operator norm. The first estimate is crude, but is of an interesting nature since a knowledge of first derivatives at a point allows estimates of the entire functions in the complex plane.

THEOREM IX. If $M(z)$ is a matrix valued entire function satisfying (2), such that $M(0)=1$, then

$$
M^{\prime}(0)=\left(\begin{array}{ll}
-\beta & \alpha \\
-\gamma & \beta
\end{array}\right),
$$

where $\alpha, \beta, \gamma$ are real numbers such that

$$
\alpha \geqq 0, \quad \gamma \geqq 0, \quad \text { and } \beta^{2} \leqq \alpha \gamma .
$$

If

$$
M(z)=\sum M_{n} z^{n}
$$

is the power series expansion, then

$$
n !\left\|M_{n}\right\| \leqq(\alpha+\gamma)^{n} .
$$

It follows from Theorem IX that the entries of $M(z)$ have exponential type. This also follows from Theorems III and IV of [8], where it is shown that they satisfy

$$
\int\left(1+t^{2}\right)^{-1} \log ^{+}|F(t)| d t<\infty
$$

ThEOREM X. In the situation of Theorem VI, let

$$
\tau(a)=\int_{0}^{a}\left[\alpha^{\prime}(t) \gamma^{\prime}(t)-\beta^{\prime}(t)^{2}\right]^{1 / 2} d t
$$

for $0<a<\infty$. If $F(z)$ denotes any one of the entire functions $A(a, z), B(a, z)$, $C(a, z), D(a, z)$, then

$$
\lim r^{-1} \log \left|F\left(r e^{i \theta}\right)\right|=\tau(a)|\sin \theta|
$$

for $\theta \neq 0, \pi$, as $r \rightarrow \infty$.

The construction of Theorem VIII has special properties when $m(t)$ satisfies (9).

THEOREM XI. Suppose that, in the situation of Theorem VI, $m(t)$ satisfies (10) and that $\alpha(t)>\alpha(0)$ for $t>0$. Then, there exists a unique nonnegative measure $\mu$ on the Borel sets of the real line such that

$$
\int\left(1+t^{2}\right)^{-1} d \mu(t)<\infty
$$


and

$$
\frac{y}{\pi} \int \frac{d \mu(t)}{(t-x)^{2}+y^{2}}=\operatorname{Re} \lim _{b \rightarrow \infty} i C(b, z) / A(b, z)
$$

for $y>0$. If $a>0$ is a regular point with respect to $m(t)$, then $\operatorname{HC}(E(a))$ is contained isometrically in $L^{2}(\mu)$. The union of such $\operatorname{se}(E(a))$, with a regular, is dense in $L^{2}(\mu)$.

THEOREM XII. If $\mu$ is a nonnegative measure on the Borel sets of the real line which satisfies (16) and does not vanish identically, then $\mu$ is of the form (17) for some choice of $m(t)$ as in Theorem XI.

Our proofs use a number of elementary properties of entire functions of genus 0 or 1 .

\section{LEMMA 1. If}

$$
E(z)=F(z) \exp \left(-a z^{2}\right)
$$

is an entire function satisfying (1) such that $a \geqq 0$ and $F(z)$ has genus 0 or 1 , then there is a sequence $\left(F_{n}(z)\right)$ of polynomials with no real zeros, satisfying (1), such that

$$
E(z)=\lim E_{n}(z)
$$

uniformly on every bounded subset of the complex plane. If $E^{*}(z)=E(-z)$ for all complex $z$, we may choose each $E_{n}(z)$ so that $E_{n}^{*}(z)=E_{n}(-z)$ for all complex $z$.

LemMa 2. Let $E(z)$ be an entire function satisfying (1). A necessary and sufficient condition that it be of the form (18), where $a \geqq 0$ and $F(z)$ has genus 0 or 1 , is that for each real $x,|E(x+i y)|$ is a nondecreasing function of $y \geqq 0$.

LEMMA 3. Let $E(z)$ be an entire function satisfying (1). A necessary and sufficient condition that $E(z)=F(z) G(z)$ where $F(z)$ has genus 0 or 1 and $G(z)$ is an entire function with only real zeros, satisfying (12), is that

$$
\int\left(1+t^{2}\right)^{-1} \operatorname{Re}\left[i E^{\prime}(t) / E(t)\right] d t<\infty .
$$

LEMMA 4. If $E(z)$ is an entire function satisfying (1), of the form (18) where $a \geqq 0$ and $F(z)$ has genus 0 or 1 , then $K(x+i y, x+i y)$ is a nondecreasing function of $y \geqq 0$ for each fixed $x$.

LEMMA 5. Let $E(z)$ be an entire function satisfying (1), of the form (18) where $a \geqq 0$ and $F(z)$ has genus 0 or 1 . If $E(0)=1$, then

$$
\log |E(z)| \leqq x A^{\prime}(0)+y B^{\prime}(0)+\left[A^{\prime}(0)^{2}-A^{\prime \prime}(0)+B^{\prime}(0)^{2}\right]|z|^{2} / 2
$$

for all complex $z$. 
LEMMA 6. Let $F(z)$ be an entire function such that $|F(z)| \leqq M(R)$ for $|z| \leqq R$. Then,

$$
|[F(z)-F(0)] / z| \leqq 2 M(R) / R
$$

for $|z| \leqq R$.

Lemma 7. If $\left(z_{n}\right)$ is a sequence of complex numbers such that $\left|z_{n}\right| \rightarrow \infty$ and $i\left(\bar{z}_{n}-z_{n}\right) \geqq 0$ for every $n$, and

$$
\sum i\left(\bar{z}_{n}-z_{n}\right)\left|z_{n}\right|^{-2}<\infty,
$$

then there exists an entire function $E(z)$ satisfying (1) such that the zeros of $E^{*}(z)$, counted according to multiplicity, are just the numbers $\left(z_{n}\right)$.

We also use an equivalent formulation of condition (4).

LEMMA 8. Let $M(b, z)$ be a matrix valued entire function satisfying (2), and let $u$ and $v$ be complex numbers which satisfy (3) and are not both zero. Then, the failure of (4) is a necessary and sufficient condition that

$$
M(b, z)=M(a, z) M(a, b, z),
$$

where $M(a, b, z)$ is a matrix valued entire function satisfying (2) and

$$
\begin{array}{ll}
A(a, z)=1-\beta z, & B(a, z)=\alpha z, \\
C(a, z)=-\gamma z, & D(a, z)=1+\beta z,
\end{array}
$$

and $\alpha, \beta, \gamma$ are real numbers, not all zero, such that

$$
\alpha \geqq 0, \quad \gamma \geqq 0, \quad \beta^{2}=\alpha \gamma, \quad \alpha v=\beta u, \quad \beta v=\gamma u .
$$

In this case, the factorization can be made so that $M(a, b, z)$ satisfies (4).

Lemma 9. If in the situation of Theorem VI, $\alpha(t)>\alpha(0)$ for $t>0$, then

$$
[B(a, z) \bar{A}(a, z)-A(a, z) \bar{B}(a, z)] /(z-\bar{z})>0,
$$

for all complex $z$. For $a>0$ and $y>0$, let $D(a, z)$ be the closed disk in the complex plane of center

$$
[D(a, z) \bar{A}(a, z)-C(a, z) \bar{B}(a, z)] /[i A(a, z) \bar{B}(a, z)-i B(a, z) \bar{A}(a, z)]
$$

and radius

$$
[i A(a, z) \bar{B}(a, z)-i B(a, z) \bar{A}(a, z)]^{-1} .
$$

Then,

$$
w \rightarrow \frac{[D(a, z)+i C(a, z)]+[D(a, z)-i C(a, z)] w}{[A(a, z)-i B(a, z)]-[A(a, z)+i B(a, z)] w}
$$

is a conformal mapping of the unit disk $|w| \leqq 1$ onto $D(a, z)$. The disk $D(a, z)$ is contained in the right half plane, $x \geqq 0$. When $a \leqq b, \mathbb{D}(a, z)$ contains $\mathbb{D}(b, z)$. 
Lemma 10. In the situation of Theorem VI, let $u$ and $v$ be complex numbers which satisfy (3) and are not both zero. Suppose that $\alpha(t)+\gamma(t)>\alpha(0)+\gamma(0)$ for $t>0$, and that $a>0$. A necessary and sufficient condition that $M(a, z)$ satisfy (4) is that

$$
\alpha(t) v \bar{v}+\gamma(t) u \bar{u}-2 \beta(t) u \bar{v}>\alpha(0) v \bar{v}+\gamma(0) u \bar{u}-2 \beta(0) u \bar{v}
$$

for $t>0$.

We need, too, a result which is closely related to previous work.

LEMMA 11. Let $E(z)$ be an entire function of exponential type with no real zeros, which satisfies (1) and (14). Let $\mu$ be a nonnegative measure on the Borel sets of the real line such that $\operatorname{JC}(E)$ is contained isometrically in $L^{2}(\mu)$. Let $G(z)$ be an entire function of exponential type such that

$$
\lim \sup |x|^{-1} \log |G(x)|=0,
$$

as $|x| \rightarrow \infty$. If $G(z)$ belongs to $L^{2}(\mu)$ and if

$$
G(i y)=o(E(i|y|)),
$$

as $|y| \rightarrow \infty$, then $G(z)$ belongs to $\mathfrak{H C}(E)$.

LемMA 12. Let $E(a, z)$ and $E(b, z)$ be entire functions with no real zeros, satisfying (1), such that $\mathfrak{H C}(E(a))$ is contained isometrically in $\operatorname{HC}(E(b))$. If $[F(z)-F(w)] /(z-w)$ belongs to $\mathfrak{H}(E(b))$ whenever $F(z)$ belongs to $\mathfrak{H C}(E(b))$, then it belongs to $\mathfrak{H C}(E(a))$ whenever $F(z)$ belongs to $\mathfrak{H C}(E(a))$.

Proof of Lemma 1. Since $F(z)$ has genus 0 or 1 ,

$$
F^{*}(z)=c z^{k} e^{b z} \prod\left[\left(1-z / z_{n}\right) \exp \left(z / z_{n}\right)\right]
$$

where $\sum\left|z_{n}\right|^{-2}<\infty$. Since $E(z)$ satisfies (1) and $a$ is real, $F(z)$ satisfies (1). In particular, $F(z)$ has no zeros for $y>0$, and therefore $i\left(\bar{z}_{n}-z_{n}\right) \geqq 0$, for every $n$. Since $F(z)$ satisfies (1), $F^{*}(z) / F(z)$ is analytic for $y>0$ and is bounded by 1 . By Boas [1, p. 85], (20) holds. Let $s$ denote the sum of this series. Then

$$
F^{*}(z) / F(z)=(c / \bar{c}) \exp [(b-b-i s) z] \prod\left[\left(1-z / z_{n}\right) /\left(1-z / \bar{z}_{n}\right)\right]
$$

for $y>0$. Since $F^{*}(z) / F(z)$ is bounded by 1 for $y>0$, so is $\exp [(b-b-i s) z]$ and

$$
s \leqq i \bar{b}-i b .
$$

The choice of a sequence $\left(E_{n}(z)\right)$ is now made from (23) as in Boas [1, pp. 230-231 ]. If $E^{*}(z)=E(-z), b+\bar{b}=0$ and the zeros of $E(z)$ are symmetrically placed about the imaginary axis. In this case, the approximating polynomials are to be chosen with their zeros symmetrically placed about the imaginary axis. Boas allows his approximating polynomials to have real zeros, but these are easily removed by small translations parallel to the imaginary axis. 
Proof of Lemma 2, the necessity. Since $a \geqq 0, \exp \left(-a z^{2}\right)$ is a nondecreasing function of $y \geqq 0$ for each fixed $x$. We prove the necessity by showing that $|F(x+i y)|$ is a nondecreasing function of $y \geqq 0$ for each fixed $x$. From (23) we have

$$
F^{\prime}(z) / F(z)=k / z+b+\sum\left[\left(z-\bar{z}_{n}\right)^{-1}+\bar{z}_{n}^{-1}\right]
$$

and therefore

$$
\operatorname{Re}\left[i F^{\prime}(z) / F(z)\right]=(i b-i b-s) / 2+k y|z|^{-2}+\sum\left(y+y_{n}\right)\left|z-\bar{z}_{n}\right|^{-2}
$$

is nonnegative for $y>0$ because of (24). The necessity follows.

Proof of Lemma 3, the necessity. Since $E(z)$ satisfies (1) and $G(z)$ satisfies (12), $F(z)$ satisfies (1). By the necessity for Lemma 2,

$$
\operatorname{Re}\left[i F^{\prime}(z) / F(z)\right] \geqq 0
$$

for $y>0$. By the Poisson representation of a function positive and harmonic in a half plane, (19) is satisfied with $E(z)$ replaced by $F(z)$. Since $G(z)$ satisfies (12),

$$
\operatorname{Re}\left[i E^{\prime}(x) / E(x)\right]=\operatorname{Re}\left[i F^{\prime}(x) / F(x)\right]
$$

and (19) follows.

Proof of Lemma 3, the sufficiency. Let $\left(z_{n}\right)$ be the nonreal zeros of $E^{*}(z)$. Let

$$
P_{n}(z)=\prod_{1}^{n}\left(z-\bar{z}_{k}\right)
$$

Then, $P_{n}(z)$ has no zeros for $y \geqq 0$ and $\left|P_{n}(\bar{z})\right| \leqq\left|P_{n}(z)\right|$ for these values of $z$. Since $E(z)$ satisfies (1),

$$
E_{n}(z)=E(z) / P_{n}(z)
$$

has no zeros for $y>0$ and $\left|E_{n}(\bar{z})\right| \leqq\left|E_{n}(z)\right|$ for $y \geqq 0$. Since $E(z)=P_{n}(z) E_{n}(z)$,

$$
\operatorname{Re}\left[i E^{\prime}(x) / E(x)\right]=\operatorname{Re}\left[i P_{n}^{\prime}(x) / P_{n}(x)\right]+\operatorname{Re}\left[i E_{n}^{\prime}(x) / E_{n}(x)\right] \text {. }
$$

Because of the last inequality,

$$
\operatorname{Re}\left[i E_{n}^{\prime}(x) / E_{n}(x)\right] \geqq 0 .
$$

A direct computation yields

$$
\int\left(1+t^{2}\right)^{-1} \operatorname{Re}\left[i P_{n}^{\prime}(t) / P_{n}(t)\right] d t=\pi \sum_{i}^{n}\left(1+y_{n}\right)\left|z_{n}+i\right|^{-2},
$$

which is at most equal to (19). By the arbitrariness of $n,(20)$ holds and $\sum\left|z_{n}\right|^{-2}<\infty$. Let $F_{0}(z)$ be defined by (23) with $c=1, k=0$, and $b$ chosen so that equality holds in (24). Then, $F_{0}(z)$ satisfies (1) or is a real exponential. 
Therefore, $G_{0}(z)=E(z) / F_{0}(z)$ is an entire function which has no nonreal zèros and $\left|G_{0}(\bar{z})\right| \leqq\left|G_{0}(z)\right|$ for $y>0$. It follows that $G_{0}^{*}(z) / G_{0}(z)$ is an entire function which has no zeros and is bounded by 1 for $y>0$, and has absolute value 1 on the real axis. By Boas [1, p. 92], this function is equal to $\exp (2 i h z)$ for some $h \geqq 0$. Let

$$
F(z)=F_{0}(z) \exp (-i h z), \quad G(z)=G_{0}(z) \exp (i h z) .
$$

It is clear that these functions have the required properties.

Proof of Lemma 2, the sufficiency. Our hypothesis implies that when $h>0, E(z-i h)$ satisfies (1). As in the proof of necessity for Lemma $3, E(z-i h)$ satisfies (20). Let $\left(z_{n}\right)$ be the nonzero zeros of $E_{n}^{*}(z)$. We have shown that

$$
\sum\left(h+y_{n}\right)\left|z_{n}+i h\right|^{-2}<\infty
$$

and this implies that $\sum\left|z_{n}\right|^{-2}<\infty$ and that (20) holds for the sequence $\left(z_{n}\right)$. Let $F_{0}(z)$ be defined by (23) with $c=1, k$ equal to the multiplicity of the zero of $E(z)$ at the origin, and $b$ chosen so that equality holds in (24). As in the proof of sufficiency for Lemma 3 , there is a real number $h \geqq 0$ such that $G(z)=E(z) / F(z)$ satisfies (12) if we define $F(z)=F_{0}(z) \exp (-i h z)$. By construction, $F(z)$ has genus 0 or 1 and satisfies (1), whereas $G(z)$ is an entire function with no zeros. By the necessity for Lemma 2, $F(z-i h)$ satisfies (1) for each $h>0$ and therefore $G(z-i h)$ satisfies (1) or (12). Since $G(z)$ satisfies (12), we see that $|G(x+i y)|$ is a nondecreasing function of $y \geqq 0$ for each fixed $x$. By the Poisson representation of a function positive and harmonic in a half plane,

$$
\operatorname{Re}\left[i G^{\prime}(z) / G(z)\right]=2 a y
$$

for $y \geqq 0$, where $a \geqq 0$, since the harmonic function on the left vanishes on the real axis because of (12). By (12), the same formula holds for all complex $z$. By the Cauchy-Riemann equations

$$
G^{\prime}(z) / G(z)+2 a z=\beta
$$

is a real constant. Therefore,

$$
G(z)=G(0) \exp \left[\beta z-a z^{2}\right] .
$$

The lemma follows on rearranging the factors of $E(z)$.

Proof of Lemma 4. By Lemma 1, it is sufficient to consider the case that $E(z)$ is a polynomial. Let $w$ be held fixed with $i(\bar{w}-w) \geqq 0$, and consider $K(w, z)$ as a polynomial in $z$. Then, (1) implies that $K(w, z)$ has no zeros for $y>0$. By Lemma $2,|K(w, w+i h)| \geqq K(w, w)$ when $h \geqq 0$. By the definition of $\operatorname{HC}(E)$,

$$
|K(w, w+i h)|^{2} \leqq\|K(w, t)\|^{2} K(w+i h, w+i h)
$$

where 


$$
\|K(w, t)\|^{2}=\langle K(w, t), K(w, t)\rangle=K(w, w) .
$$

The two inequalities imply that

$$
K(w+i h, w+i h) \geqq K(w, w) .
$$

Proof of Lemma 5. By Lemma 1, it is sufficient to prove the lemma in the case that $E(z)$ is a polynomial. Then,

$$
E(z)=\prod\left(1-z / \bar{z}_{n}\right)
$$

with only a finite number of factors, and $i\left(\bar{z}_{n}-z_{n}\right) \geqq 0$ for every $n$. From this we find $\left(z_{n}=x_{n}+i y_{n}\right)$

$$
\begin{aligned}
& B^{\prime}(0)=\sum y_{n}\left|z_{n}\right|^{-2}, \quad-A^{\prime}(0)=\sum x_{n}\left|z_{n}\right|^{-2} \\
& A^{\prime}(0)^{2}-A^{\prime \prime}(0)-B^{\prime}(0)^{2}=\sum\left(x_{n}^{2}-y_{n}^{2}\right)\left|z_{n}\right|^{-4}
\end{aligned}
$$

and so

$$
A^{\prime}(0)^{2}-A^{\prime \prime}(0)+B^{\prime}(0)^{2} \geqq \sum\left|z_{n}\right|^{-2} .
$$

The inequality $\log t \leqq t-1$ for $t>0$ becomes

$$
\log \left|1-z / z_{n}\right| \leqq\left[-x x_{n}+y y_{n}+|z|^{2} / 2\right]\left|z_{n}\right|^{-2}
$$

when $t=\left|1-z / \bar{z}_{n}\right|^{2}$. The desired inequality follows on summing over $n$ and making the obvious substitutions.

Proof of Lemma 6. Apply the maximum principle to $[F(z)-F(0)] / z$.

Proof of Lemma 7. By the Weierstrass factorization, there exists an entire function $F(z)$ whose zeros are the given ones, counting multiplicities. Then, $G(z)=F(z) F^{*}(z)$ is an entire function. By Boas [1, p. 90], (20) implies that

$$
B(z)=\prod\left[\left(1-z / z_{n}\right) /\left(1-z / \bar{z}_{n}\right)\right]
$$

converges uniformly on any bounded set at a positive distance from the zeros of $G(z)$. Obviously, $B(z) B^{*}(z)=1$ and $|B(z)|<1$ for $y>0$. Therefore, $G(z) / B(z)$ is an entire function satisfying (1) and all its zeros have even multiplicity. We may write $G(z) / B(z)=E(z)^{2}$ for some entire function $E(z)$. It satisfies (1) and has the desired zeros.

Proof of Lemma 8. Let

$$
|u+i v|^{2} M(z)=\left(\begin{array}{rr}
v & -u \\
u & v
\end{array}\right) M(b, z)\left(\begin{array}{rr}
\bar{v} & \bar{u} \\
-\bar{u} & \bar{v}
\end{array}\right) .
$$

It is easily verified that $M(z)$ is a matrix valued entire function satisfying (2), and that $M(b, z)$ satisfies (4) for the given $u, v$ if, and only if, $M(z)$ satisfies (4) for $u=0, v=1$. For the rest of the proof, we will restrict ourselves to the case $u=0, v=1$, since the general case can be obtained by this simple inner automorphism. The hypothesis $(2)$ for $M(b, z)$ implies that 


$$
f(z)=[D(b, z)+i C(b, z)] /[A(b, z)-i B(b, z)]
$$

is defined and analytic for $y>0$ and has a nonnegative real part. This function remains continuous in the closed half plane and

$$
\operatorname{Re} f(x)=|A(b, x)-i B(b, x)|^{-2} .
$$

By the Poisson representation, there is a number $k \geqq 0$ such that

$$
\operatorname{Re} f(z)=\frac{y}{\pi} \int \frac{\operatorname{Re} f(t) d t}{(t-x)^{2}+y^{2}}+k y
$$

for $y>0$. By the Lebesgue dominated convergence theorem,

$$
k=\lim y^{-1} \operatorname{Re} f(i y)
$$

as $y \rightarrow+\infty$. Furthermore,

$$
\operatorname{Re}[f(z)+i k z] \geqq 0
$$

for $y>0$. If $M(b, z)$ does not satisfy (4) for $u=0, v=1$, then $k>0$. Let

$$
A(a, b, z)=A(b, z), \quad B(a, b, z)=B(b, z) .
$$

By Theorem IV of [8], there exist entire functions $C(a, b, z), D(a, b, z)$ such that $M(a, b, z)$ so defined satisfies (2) and (4) for $u=0, v=1$. By adding constant multiples of $A(a, b, z)$ and $B(a, b, z)$ to $C(a, b, z)$ and $D(a, b, z)$, if necessary, we may suppose these two functions chosen so that

$$
C(a, b, 0)=C(b, 0), \quad D(a, b, 0)=D(b, 0) .
$$

Then, (2) implies that

$$
g(z)=[D(a, b, z)+i C(a, b, z)] /[A(a, b, z)-i B(a, b, z)]
$$

is defined and analytic for $y>0$ and has a nonnegative real part. By construction, $\operatorname{Re} g(x)=\operatorname{Re} f(x)$ and $g(i y)=0(y)$ as $y \rightarrow+\infty$. By the Poisson representation, $g(z)$ and $f(z)+i k z$ have the same real part for $y>0$. By the CauchyRiemann equations, these two functions differ by a constant in the upper half plane. Since they are continuous in the closed half plane and agree at the origin, they are identical. It follows that

$$
D(b, z)+i C(b, z)+i k A(b, z)+k B(b, z)=D(a, b, z)+i C(a, b, z)
$$

for $y>0$, and hence by analyticity, for all complex $z$. Since we have entire functions which are real for real $z$,

$$
\begin{aligned}
& C(a, b, z)=C(b, z)+k A(b, z), \\
& D(a, b, z)=D(b, z)+k B(b, z),
\end{aligned}
$$

for all complex $z$. Let $M(a, z)$ be defined as in the statement of the theorem with $\alpha=0, \beta=0, \gamma=k$. The sufficiency then follows. Since the necessity is 
proved by essentially reversing the above procedure, the proof is omitted. It was clear from the construction that $M(a, b, z)$ satisfies (4) with $u=0, v=1$.

Proof of Theorem VI. The theorem is Theorem XA of [8] with $C_{0}=1$, $S_{0}=0, C_{1}=1, S_{1}=0$,

$$
\begin{gathered}
C_{0}(t, z)=A(t, z), \quad S_{0}(t, z)=B(t, z), \\
-S_{1}(t, z)=C(t, z), \quad C_{1}(t, z)=D(t, z), \\
a(t)=\gamma^{\prime}(t), \quad b(t)=\beta^{\prime}(t), \quad c(t)=\alpha^{\prime}(t) .
\end{gathered}
$$

Proof of Lemma 9. It follows from (2) that $E(a, z)$ is without zeros for $y \geqq 0$ and that $|E(a, \bar{z})| \leqq|E(a, z)|$ for these values of $z$. In other words,

$$
\left|E^{*}(a, z) / E(a, z)\right| \leqq 1,
$$

for $y>0$. By Boas [1, p. 92], we must have both of these inequalities strict unless $E^{*}(a, z) / E(a, z)$ is a constant. In that case, $E(a, z)$ satisfies (12) and has no zeros. Since $E(a, z)$ has exponential type and satisfies (14), it is then a constant, which is 1 by its value at the origin. If $a>0$, the $z$ derivative of $B(a, z)$ at the origin is $\alpha(a)-\alpha(0)$, which is positive by hypothesis. Therefore, $E(a, z)$ satisfies $(1)$ in this case and $K(a, z, z)>0$ for all complex $z$. When $a<b$, let $M(a, b, z)$ be defined by (26). The conclusions about conformal mapping follow from (2) and (6) using Lemma 1 of [8].

Proof of Lemma 10. The inner automorphism in the proof of Lemma 8 will reduce the problem to the case $u=0, v=1$, and we restrict ourselves to this choice of $u$ and $v$. By (7), $\alpha(t)$ is a nondecreasing function of $t \geqq 0$. Therefore, if $\alpha(a)=\alpha(0)$ for some $a>0, \alpha(t)=\alpha(0)$ for $0 \leqq t \leqq a$. Since (7) implies that

$$
[\beta(t)-\beta(0)]^{2} \leqq[\alpha(t)-\alpha(0)][\gamma(t)-\gamma(0)],
$$

it follows that $\beta(t)=\beta(0)$ for $0 \leqq t \leqq a$. Definition (13) then yields

$$
\begin{array}{ll}
A(t, z)=1, & B(t, z)=0, \\
C(t, z)=\gamma(0) z-\gamma(t) z, & D(t, z)=1,
\end{array}
$$

when $0 \leqq t \leqq a$, and for $t \geqq a$,

$$
M(t, z)=M(a, z) M(a, t, z),
$$

where $M(a, t, z)$ is a matrix valued entire function of $z$ which satisfies (2). Since $\alpha(t)+\gamma(t)>\alpha(0)+\gamma(0)$ for $t>0$ by hypothesis, $\gamma(t)>\gamma(0)$ for $0<t \leqq a$. By Lemma $8, M(t, z)$ does not satisfy (4) for $u=0, v=1$, when $t>0$. Conversely, suppose that (21) is satisfied for $t>0$. Let

$$
f(a, z)=[D(a, z)+i C(a, z)] /[A(a, z)-i B(a, z)] .
$$

As in the proof of Lemma 8,

$$
k(a)=\lim y^{-1} \operatorname{Re} f(a, i y)
$$


exists as $y \rightarrow+\infty$. In the notation of Lemma $9, f(a, i y)$ and $f(b, i y)$ belong to $D(a, i y)$ when $a \leqq b$. By Lemma 4 , the radius of $D(a, i y)$ remains bounded as $y \rightarrow+\infty$. Therefore, $k=k(a)=k(b)$ is a constant. On the other hand, we see from the proof of Lemma 8 that

$$
\left(\begin{array}{ll}
1 & 0 \\
k z & 1
\end{array}\right) M(a, z)
$$

is a matrix valued entire function which satisfies (2). But (2) implies positivity properties of the derivatives of the entire functions at the origin. These are stated in Theorem IX, but the proof is immediate. They imply that

$$
0 \leqq k \leqq \gamma(a)-\gamma(0) .
$$

By (9) and the arbitrariness of $a, k=0$. As in the proof of Lemma 8, it follows that $M(a, z)$ satisfies (4) for $u=0, v=1$.

Proof of Lemma 11. Let $L$ be an element of $L^{2}(\mu)$ which is orthogonal to $\operatorname{JC}(E)$ and consider

$$
\begin{aligned}
& P(z)=\int \frac{E(z) G(t)-G(z) E(t)}{t-z} \bar{L}(t) d \mu(t), \\
& Q(z)=\int \frac{E^{*}(z) G(t)-G(z) E^{*}(t)}{t-z} \bar{L}(t) d \mu(t) .
\end{aligned}
$$

Our hypotheses make these integrals absolutely convergent for all complex $z$. The argument of $[2, \mathrm{p} .147]$ shows that $P(z)$ and $Q(z)$ are entire functions of exponential type. By Boas [1, p. 97], the indicator diagrams of $E(z)$ and $G(z)$ are vertical segments, and the same is true of $P(z)$ and $Q(z)$ by the estimates of [2]. Since $L$ is orthogonal to $\mathfrak{H}(E)$,

$$
P(z) E^{*}(z)-Q(z) E(z)=0
$$

vanishes identically. Since $E(z)$ has no real zeros by hypothesis and satisfies (1), there is an entire function $H(z)$ such that

$$
P(z)=E(z) H(z), \quad Q(z)=E^{*}(z) H(z),
$$

for all complex z. As a result of the representation theorem, Boas [1, p. 92], applied to $E(z), H(z)$ has exponential type and its indicator diagram is a vertical line segment. By the Lebesgue dominated convergence theorem and (22), $H(z)$ goes to zero at both ends of the imaginary axis. By Boas [1, pp. 83, 97], $H(z)$ vanishes identically. By the arbitrariness of $L$,

$$
\begin{aligned}
& {[E(z) G(w)-G(z) E(w)] /(z-w),} \\
& {\left[E^{*}(z) G(w)-G(z) E^{*}(w)\right] /(z-w),}
\end{aligned}
$$

belong to $\operatorname{se}(E)$ for every complex number $w$. Let 


$$
G(z)=[G(z)-S(z)]+S(z),
$$

where $G(z)-S(z)$ belongs to $\mathfrak{H C}(E)$ and $S(z)$ is orthogonal to $\mathfrak{H C}(E)$. Then,

$$
\begin{aligned}
& {[E(z) S(w)-S(z) E(w)] /(z-w),} \\
& {\left[E^{*}(z) S(w)-S(z) E^{*}(w)\right] /(z-w),}
\end{aligned}
$$

belong to $\mathfrak{H C}(E)$ for every complex number $w$. The orthogonality of $S(z)$ to $\mathfrak{H C}(E)$ implies that for every nonreal complex number $w$,

$$
\begin{gathered}
\langle[S(t) K(w, w)-K(w, t) S(w)](t-\bar{w}) /(t-w), \\
[S(t) K(\bar{w}, \bar{w})-K(\bar{w}, t) S(\bar{w})]\rangle \\
\quad=\|S\|^{2} K(w, w)+|S(\bar{w})|^{2} K(w, w),
\end{gathered}
$$

which is equal to the product of

$$
\|S(t) K(w, w)-K(w, t) S(w)\|
$$

and

$$
\|S(t) K(\bar{w}, \bar{w})-\mathrm{K}(\bar{w}, t) S(\bar{w})\|
$$

with the inner product taken in $L^{2}(\mu)$. In particular, $|S(\bar{w})|=|S(w)|$. Equality occurs in the Schwarz inequality only when there is linear dependence. So,

$$
[S(z) K(w, w)-K(w, z) S(w)](z-\bar{w}) /(z-w)
$$

and

$$
S(z) K(\bar{w}, \bar{w})-K(\bar{w}, z) S(\bar{w})
$$

are linearly dependent. Therefore, $S(z)$ satisfies (25) with $G(z)$ replaced by $S(z)$. Since $S(z)$ is orthogonal to $\mathfrak{F C}(E)$, it follows that there exist complex numbers $u$ and $v$ such that $S(z)$ satisfies (5). Since $|S(\bar{z})|=|S(z)|$ for all complex $z, u$ and $v$ also satisfy (3). Therefore, there is a real number $\alpha$ such that

$$
u \cos \alpha+v \sin \alpha=0,
$$

and $G(z)$ is a constant multiple of $e^{i \alpha} E(z)-e^{-i \alpha} E^{*}(z)$, which does not belong to $\mathfrak{F C}(E)$. We are now in the situation of [6]. Since $G(z)$ satisfies (22) by hypothesis and $G(z)-S(z)$ satisfies $(22)$ by the definition of $\mathfrak{H C}(E), S(z)$ satisfies (22). Therefore,

$$
\int\left|\frac{S(t)}{E(t)}\right|^{2} d t=2 \pi i \sum \frac{|S(t)|^{2}}{E(t) \bar{E}^{\prime}(t)-\bar{E}(t) E^{\prime}(t)}
$$

where on the right $t$ ranges in the real numbers such that $e^{i \alpha} E(t)$ is real. By construction, $S(t)$ vanishes for all such $t$. By the integral on the left, $S(z)$ vanishes for all real $z$ and, by analyticity, for all complex $z$. The theorem follows. 
Proof of Lemma 12. By Theorem III of [8], $E(b, z)$ is an entire function of exponential type which satisfies (14). Since $\mathfrak{H C}(E(a))$ is contained isometrically in $\mathfrak{H}(E(b)), K(a, z, z) \leqq K(b, z, z)$ for all complex $z$. It follows that $E(a, z)$ has exponential type and satisfies (14). Let $\mu$ be the nonnegative measure on the Borel sets of the real line defined by

$$
\mu(S)=\int_{S}|E(b, t)|^{-2} d t
$$

for every Borel set $S$. By definition, $\mathfrak{F C}(E(b))$ is contained isometrically in $L^{2}(\mu)$, and hence so is $\mathfrak{H C}(E(a))$ by hypothesis. If $F(z)$ belongs to $\mathfrak{F C}(E(a))$, $[F(z)-F(w)] /(z-w)$ belongs to $\mathcal{H C}(E(b))$ by hypothesis and hence is in $L^{2}(\mu)$. Since $F(z)$ is in $\operatorname{HC}(E(a))$, it satisfies (14), and (22) for $E(z)=E(a, z)$ by the definition of $\mathfrak{F}(E)$. Since $E(a, z)$ has exponential type, it has genus 0 or 1 . By Lemma $2,|E(a, i y)|$ is a nondecreasing function of $y \geqq 0$. It follows that $[F(z)-F(w)] /(z-w)$ satisfies $(22)$ for $E(z)=E(a, z)$. By Lemma 11 , this function belongs to $\mathcal{H C}(E(a))$.

Proof of Theorem I. If $G(z)$ belongs to $3 C(E)$, then (22) holds by the definition of $\mathcal{H}(E)$, and this implies (3). Therefore, there is a real number $\alpha$ such that

$$
u \cos \alpha+v \sin \alpha=0
$$

and $G(z)$ is a constant multiple of $e^{i \alpha} E(z)-e^{-i \alpha} E^{*}(z)$. By Theorem II of [8], the orthogonal complement of $G(z)$ in $\mathcal{H}(E)$ satisfies (H1), (H2), and (H3). By the proof of that theorem,

(25) $K(w, z) G(w)-K(w, w) G(z)=[K(\bar{w}, z) G(\bar{w})-K(\bar{w}, \bar{w}) G(z)](z-w) /(z-\bar{w})$

holds for all complex $z$ if $w$ is not real. Suppose that $F(z)$ and $z F(z)$ are in $\mathfrak{F}(E)$. Then, $(z-w) F(z)$ and $(z-\bar{w}) F(z)$ are in $\mathcal{H}(E)$. Take the inner product of each side of (25) with $(z-w) F(z)$, use (H1) on the right, and expand using the definition of $K(w, z)$. Then, (25) is equivalent to

$$
K(w, w)\langle(t-w) F(t), G(t)\rangle=K(\bar{w}, \bar{w})\langle(t-\bar{w}) F(t), G(t)\rangle .
$$

Since $K(\bar{w}, \bar{w})=K(w, w)>0$ and $w$ is not real, it follows that $G(z)$ is orthogonal to $F(z)$. Conversely, if the elements $F(z)$, whose product by $z$ belongs to $\mathfrak{H C}(E)$, are not dense in $\mathfrak{F C}(E)$, there is a nonzero element $G(z)$ of $\mathcal{H}(E)$ orthogonal to them all. The last identity must hold for each such $F(z)$, and this implies that each side of (25) has the same inner product with $(z-w) F(z)$. Since both sides of (25) vanish when $z=w$, it follows from the arbitrariness of $F(z)$ that (25) holds. Obviously, $G(z)$ is not the constant multiple of any $K(w, z)$. As in the proof of Theorem II of [8], it follows that (5) holds for some choice of $u$ and $v$, not both zero, since $G(z)$ does not vanish identically. By Lemma 7 of [7], $G(z)$ is then unique within a constant factor.

Proof of Theorem II. This is Theorem VII of [8] with 


$$
\begin{aligned}
C_{a}(z) & =A(a, z), & & S_{a}(z)=B(a, z), \\
C_{b}(z) & =A(b, z), & & S_{b}(z)=B(b, z), \\
C_{0}(z) & =A(a, b, z), & & S_{0}(z)=B(a, b, z), \\
-S_{1}(z) & =C(a, b, z), & & C_{1}(z)=D(a, b, z) .
\end{aligned}
$$

Proof of Theorem III. This is Theorem VIII of [8] except for the uniqueness of $M(a, b, z)$, or in other words, of $C_{0}(z), S_{0}(z), C_{1}(z), S_{1}(z)$ in the notation of [8]. This will follow as soon as it is shown that in the situation of Theorem VII of [8], formulas (32), (33), (34), and (35) of [8] must hold with $E(z)$ $=E_{b}(z)$ and $E_{c}(z)$ defined by formula (12) of [8]. Since the proofs are essentially the same as that of formula (21) of [8], they are omitted.

Proof of Theorem IV. For each $a>0$ and each complex number $w$, let $M(a, t, w)$ be the unique continuous function of $t \geqq a$ such that

$$
M(a, b, w) I-I=\int_{a}^{b} M(a, t, w) d m(t)
$$

for $b \geqq a$. The existence of $M(a, b, z)$ is Theorem VI, which has already been established, with a change of variable. For each fixed $a$ and $b, M(a, b, z)$ is a matrix valued entire function of $z$ which satisfies (2).

We start by showing uniqueness. Let $\left(E_{i}(t, z)\right)$ be two families of entire functions, $i=1,2$, which satisfy (1) for each fixed $t$ and which satisfy (11) for each fixed $z=w$. By the uniqueness part of Theorem VI, already proved, (6) must hold whenever $a<b$, for each family. It follows that

$$
B_{1}(b, z) A_{2}(b, z)-A_{1}(b, z) B_{2}(b, z)=B_{1}(a, z) A_{2}(a, z)-A_{1}(a, z) B_{2}(a, z)
$$

for all complex $z$. Let this entire function be denoted by $F(z)$. It is independent of $a$ and $b$. If the initial conditions for (11) are satisfied by both families,

$$
\lim F(z) \exp [2 \beta(t) z]=0
$$

for all complex $z$, as $t \rightarrow 0$. Therefore, $F(z)$ vanishes identically. It follows that $E_{2}(t, z) / E_{1}(t, z)$ is independent of $t$ for each fixed $z$ with $y>0$. Our initial conditions imply that this ratio is 1 . So, $E_{2}(t, z)=E_{1}(t, z)$ for $y>0$, and hence by analyticity, for all complex $z$. Now, let us prove existence.

Let $E(a, b, z)=A(a, b, z)-i B(a, b, z)$. Since $E(a, b, 0)=1$, Lemma 5 yields the estimate

$$
\begin{aligned}
\log |E(a, b, z)| \leqq & x[\beta(a)-\beta(b)]+y[\alpha(b)-\alpha(a)] \\
& +|z|^{2}[\alpha(b)-\alpha(a)]^{2} / 2+|z|^{2} \int_{a}^{b} \alpha(t) d \gamma(t),
\end{aligned}
$$

for all complex $z$. Let $b>0$ be held fixed. By (8), the entire functions

$$
E(a, b, z) \exp [-\beta(a) z]
$$


$0<a \leqq b$, are uniformly bounded on each bounded subset of the complex plane. Therefore, there is a sequence $a_{n} \backslash 0$ such that

$$
E(b, z)=\lim E\left(a_{n}, b, z\right) \exp \left[-\beta\left(a_{n}\right) z\right]
$$

exists uniformly on every bounded subset of the complex plane. Since

$$
M(a, c, z)=M(a, b, z) M(b, c, z)
$$

when $c \geqq b$,

$$
E(c, z)=\lim \} E\left(a_{n}, c, z\right) \exp \left[-\beta\left(a_{n}\right) z\right]
$$

exists uniformly on bounded subsets of the complex plane when $c \geqq b$, and a similar argument shows that this is true also when $c \leqq b$. Since $E(c, z)$ is a limit of entire functions satisfying (1), it has no zeros for $y>0$ and $|E(c, \bar{z})|$ $\leqq|E(c, z)|$ for these values of $z$. Therefore, it satisfies (1) or (12), and this last possibility is ruled out because

$$
B^{\prime}(c, 0)=\alpha(c)-\alpha(0)>0
$$

by hypothesis. Our definition implies that (6) holds whenever $a<b$. Therefore, (11) holds for each complex number $w$. From (27) we obtain the estimate

$$
\begin{aligned}
\log |E(c, z)| \leqq & x \beta(c)+y[\alpha(c)-\alpha(0)] \\
& +|z|^{2}[\alpha(c)-\alpha(0)]^{2} / 2+|z|^{2} \int_{0}^{c} \alpha(t) d \gamma(t) .
\end{aligned}
$$

By (8) and Lemma 6,

$$
\lim E(c, z) \exp [\beta(c) z]=1,
$$

as $c \rightarrow 0$. This completes the proof of existence. By the uniqueness, the definition is independent of the defining sequence $\left(a_{n}\right)$. Therefore,

$$
E(b, z)=\lim E(a, b, z) \exp [-\beta(a) z]
$$

uniformly on bounded sets, as $a \rightarrow 0$.

The function $E(b, z)$ has no real zero $w$, since otherwise (6) would imply that $E(a, w)=0$ whenever $a<b$ and this contradicts the initial conditions for (11). Since $E(b, z)$ is the limit of entire functions of genus 0 or 1 , Lemma 2 implies that $|E(b, x+i y)|$ is a nondecreasing function of $y \geqq 0$ for each fixed $x$. Therefore,

$$
E(b, z)=F(b, z) \exp \left[-k(b) z^{2}\right]
$$

where $F(b, z)$ has genus 0 or 1 and $k(b) \geqq 0$. But, (6) implies that $k=k(b)$ is independent of $b$ and the initial conditions for (11) make $k=0$. So, $E(b, z)$ has genus 0 or 1 .

Now, let us show that when $b<c$ are regular points with respect to $m(t)$, $\mathfrak{H C}(E(b))$ is contained isometrically in $\mathfrak{H C}(E(c))$. First, consider the case in 
which there is an interval $(a, b)$ to the left of $b$ which contains only singular points with respect to $m(t)$ and in which $m(t)$ is not a constant. Since $\alpha^{\prime}(t)$, $\beta^{\prime}(t), \gamma^{\prime}(t)$ are equivalent in $(a, b)$ to constant multiples of a single function, there are complex numbers $u$ and $v$, not both zero, satisf ying (3), such that

$$
\begin{array}{ll}
A(a, t, z)=1-\beta(t) z+\beta(a) z, & B(a, t, z)=\alpha(t) z-\alpha(a) z, \\
C(a, t, z)=\gamma(a) z-\gamma(t) z, & D(a, t, z)=1+\beta(t) z-\beta(a) z,
\end{array}
$$

for $a \leqq t \leqq b$, where

$$
\begin{aligned}
& {[\alpha(t)-\alpha(a)] v=[\beta(t)-\beta(a)] u,} \\
& {[\beta(t)-\beta(a)] v=[\gamma(t)-\gamma(a)] u .}
\end{aligned}
$$

Let $G(z)$ be defined by (5) for $E(z)=E(a, z)$, or equivalently, for $E(z)=E(b, z)$. By (6), we may choose $u$ and $v$ so that

$$
K(b, w, z)=K(a, w, z)+G(z) G^{*}(w)
$$

for all complex $z$ and $w$. Since $K(a, z, z) \geqq 0$ for all complex $z$, (22) holds for $E(z)=E(b, z)$, and this implies that

$$
\lim E^{*}(b, i y) / E(b, i y)=-(u+i v) /(u-i v),
$$

as $y \rightarrow+\infty$. Since $b$ is a regular point with respect to $m(t)$ by hypothesis, (21) is satisfied for $t \geqq b$ if 0 is replaced by $b$. By Lemma $10, M(b, c, z)$ satisfies (4) for the above $u, v$. By the proof of Theorem VII of [8], $\mathcal{F}(E(b))$ is contained isometrically in $\mathfrak{H C}(E(c))$.

If there is no interval $(b, b+E)$ to the right of $b$ containing only singular points with respect to $m(t)$, Lemma 10 shows that $M(b, c, z)$ satisfies (4) no matter what the choice of $u$ and $v$. By Theorem II, $\operatorname{Fc}(E(b))$ is contained isometrically in $\operatorname{HC}(E(c))$ in this case.

In the remaining case, there is a sequence $b_{n} \nearrow b$ for which the isometric inclusion of $\mathfrak{H C}\left(E\left(b_{n}\right)\right)$ in $\mathfrak{H C}(E(c))$ has been shown for every $n$. Then,

$$
K\left(b_{n}, w, z\right) \rightarrow K(b, w, z),
$$

for all complex $z$ and $w$. Using inner products in $\mathfrak{H C}(E(c))$, one finds that for each fixed $w$, the sequence on the left is a Cauchy sequence, and that the convergence takes place in the metric of $\mathcal{F}(E(c))$. Therefore, $K(b, w, z)$ belongs to $\operatorname{FC}(E(c))$ and

$$
K\left(b, w_{1}, w_{2}\right)=\left\langle K\left(b, w_{1}, t\right), K\left(b, w_{2}, t\right)\right\rangle
$$

for all complex $w_{1}$ and $w_{2}$, if the inner product is taken in $\mathcal{H C}(E(c))$. Therefore, the finite linear combinations of the functions $K(b, w, z)$, where $w$ ranges in the complex plane, belong to $\mathfrak{F}(E(c))$ and have the same norm there as in $\mathfrak{H C}(E(b))$. Since such combinations are dense in $\mathfrak{H C}(E(b)), \mathfrak{H}(E(b))$ is contained isometrically in $\operatorname{se}(E(c))$. 
If $a$ and $b$ are regular points with respect to $m(t)$ and if the interval $(a, b)$ contains only singular points, and if $m(t)$ is not a constant in $(a, b)$, formula (29) shows that $G(z)$ spans the orthogonal complement of $\operatorname{HC}(E(a))$ in $\operatorname{HC}(E(b))$.

Proof of Theorem VII, when $M(z)$ is a polynomial. By inspection there exist complex numbers $u$ and $v$, not both zero, satisfying (3), such that $M(z)$ does not satisfy (4) for this choice of $u$ and $v$. By Lemma 8 ,

$$
M(z)=\left(\begin{array}{cc}
1-\beta_{1} z & \alpha_{1} z \\
-\gamma_{1} z & 1+\beta_{1} z
\end{array}\right) M_{1}(z),
$$

where $\alpha_{1}, \beta_{1}, \gamma_{1}$ are real numbers, not all zero, such that

$$
\alpha_{1} \geqq 0, \quad \gamma_{1} \geqq 0, \quad \beta_{1}^{2}=\alpha_{1} \gamma_{1},
$$

and $M_{1}(z)$ is a matrix valued polynomial satisfying (2). In fact, if $M_{1}(z)$ is chosen so as to satisfy (4) for the above $u$ and $v$, the degree of $M_{1}(z)$ will be less than the degree of $M(z)$. If $M(z)$ has degree $r$, we may continue inductively to find that

$$
M(z)=\prod_{1}^{r}\left(\begin{array}{cc}
1-\beta_{k} z & \alpha_{k} z \\
-\gamma_{k} z & 1+\beta_{k} z
\end{array}\right) M(0)
$$

with the factors taken from left to right in numerical order, where $\alpha_{k}, \beta_{k}, \gamma_{k}$ are real numbers such that

$$
\begin{gathered}
\alpha_{k} \geqq 0, \quad \gamma_{k} \geqq 0, \quad \beta_{k}^{2}=\alpha_{k} \gamma_{k}, \\
\alpha_{k} \gamma_{k-1}+\gamma_{k} \alpha_{k-1}-2 \beta_{k} \beta_{k-1}>0,
\end{gathered}
$$

for $k=2, \cdots, r$. Since $M(0)=1$ by hypothesis, this factor may be omitted in (31). Let

$$
\begin{aligned}
& \alpha\left(t_{k}\right)=\alpha_{1}+\cdots+\alpha_{k}, \\
& \beta\left(t_{k}\right)=\beta_{1}+\cdots+\beta_{k}, \\
& \gamma\left(t_{k}\right)=\gamma_{1}+\cdots+\gamma_{k},
\end{aligned}
$$

for

$$
t_{k}=\alpha_{1}+\cdots+\alpha_{k}+\gamma_{1}+\cdots+\gamma_{k}
$$

and $k=0, \cdots, r$; when $k=0$, each sum is interpreted as 0 . Let $\alpha, \beta, \gamma$ be defined linearly in each interval $\left[t_{k-1}, t_{k}\right], k=1, \cdots, r$, and be constant in $\left[t_{r}, \infty\right)$. It is easily verified that $m(t)$ so defined satisfies (7), (8), and (9), and that $M(z)=M\left(t_{r}, z\right)$ when $M(t, z)$ is defined by (13).

Proof of Theorem V, when $E(z)$ is a polynomial. Since $E(z)$ satisfies (1), it does not vanish identically. Because of the factor of $G(z)$ in the formula we wish to obtain, we may restrict ourselves to the case that $E(z)$ has no real zeros and $E(0)=1$. Then, 1 belongs to $\mathcal{H C}(E)$ and therefore $[F(z)-F(w)] /(z-w)$ 
belongs to $\mathfrak{F}(E)$ whenever $F(z)$ belongs to $\mathcal{F}(E)$. By Theorem III and IV of [8], there exist entire functions $C(z)$ and $D(z)$ such that $M(z)$ so defined satisfies (2), and (4) with $u=0, v=1$. By altering $C(z)$ and $D(z)$ by constant multiples of $A(z)$ and $B(z)$, if necessary, these may be chosen so that $M(0)=1$. The proof of Theorem IV of [8] shows that $C(z)$ and $D(z)$ are polynomials. By Theorem VII, which has been established for polynomials, there is a matrix valued function $m(t)$ satisfying (7), (8), and (9), such that $M(z)$ $=M(a, z)$ for some $a \geqq 0$. We will choose $m(t)$ so that $m(0)=0$ and $m(t)$ is not constant in any interval to the right of the origin. Since $M(z)$ satisfies (4) for $u=0, v=1, \alpha(t)>\alpha(0)$ for $t>0$ by Lemma 10 . Since $E(z)$ is not a constant by hypothesis, $a>0$. The theorem now follows since (13) implies (11) when $\beta(0)=0$. If $E^{*}(z)=E(-z), A(z)$ is even and $B(z)$ is odd. The proof of Theorem IV of [8] shows that $C(z)$ is odd and $D(z)$ is even. In our proof of Theorem VII for polynomials $M(z)$, each $\beta_{k}$ vanishes, and so $\beta(t)$ vanishes identically.

Proof of Theorem V. By the choice of $G(z)$ in the desired factorization, we may restrict ourselves to the case that $E(z)$ has no real zeros and $E(0)=1$. By Lemma 1 , there is a sequence $\left(E_{n}(z)\right)$ of entire functions such that $E_{n}(z) \rightarrow E(z)$ uniformly on bounded sets and these conditions are satisfied: each $E_{n}(z)$ satisfies the hypotheses of the theorem, has no real zeros, has value 1 at the origin, and the conclusion of the theorem has already been established for this function; that is, each $E_{n}(z)$ is equal to $E_{n}\left(c_{n}, z\right)$ for some $c_{n}>0$, where $E_{n}(t, z)$ is defined by (11) for some matrix valued function $m_{n}(t)$ satisfying (7) and (8) and such that $\alpha_{n}(t)>\alpha_{n}(0)$ for $t>0$. By a reparametrization, we may suppose each $m_{n}(t)$ so chosen that $\alpha_{n}(0)=0$ and

$$
\alpha_{n}(t)+\int_{0}^{t} \alpha_{n}(s) d \gamma_{n}(s)=t
$$

for $t \geqq 0$. Then,

$$
\begin{aligned}
& A_{n}^{\prime}(0)=-\beta_{n}\left(c_{n}\right), \quad B_{n}^{\prime}(0)=\alpha_{n}\left(c_{n}\right), \\
& A_{n}^{\prime}(0)^{2}-A_{n}^{\prime \prime}(0)=2 \int_{0}^{c_{n}} \alpha_{n}(t) d \gamma_{n}(t) .
\end{aligned}
$$

Since $E_{n}(z) \rightarrow E(z)$ uniformly on bounded sets,

$$
A_{n}^{\prime}(0) \rightarrow A^{\prime}(0), \quad B_{n}^{\prime}(0) \rightarrow B^{\prime}(0), \quad A_{n}^{\prime \prime}(0) \rightarrow A^{\prime \prime}(0) .
$$

Therefore, $c=\lim c_{n}$ exists. This number is strictly positive because it is no less than $B^{\prime}(0)^{2} ; B^{\prime}(0)>0$ because $E(z)$ satisfies (1) and $E(0) \neq 0$. By (32), the functions $\left(\alpha_{n}(t)\right)$ are uniformly bounded and equicontinuous on every bounded set. We may suppose by going to a subsequence that $\alpha(t)=\lim \alpha_{n}(t)$ exists uniformly on every bounded set. Obviously, $\alpha(t)$ is a nondecreasing, absolutely continuous function such that $\alpha(0)=0$ and $\alpha(c)=B^{\prime}(0)>0$. Let $\delta$ be 
the largest value of $t$ such that $\alpha(t)=0$. When $\delta<a<b,(7)$ and (32) imply that

$$
\begin{aligned}
& 0 \leqq \gamma_{n}(b)-\gamma_{n}(a) \leqq(b-a) \alpha_{n}(a)^{-1}, \\
& \left|\beta_{n}(b)-\beta_{n}(a)\right| \leqq(b-a) \alpha_{n}(a)^{-1 / 2} \text {. }
\end{aligned}
$$

By altering each $\gamma_{n}(t)$ by a constant and by going to a subsequence, we may suppose that

$$
\beta(t)=\lim \beta_{n}(t), \quad \gamma(t)=\lim \gamma_{n}(t)
$$

exists uniformly in each interval $[a, b]$ to the right of $\delta$. Then, $m(t)$ so defined satisfies ( 7 ) for $t>\delta$. When $\delta<a<b$,

$$
\alpha_{n}(b)-\alpha_{n}(a)+\int_{a}^{b} \alpha_{n}(t) d \gamma_{n}(t)=b-a,
$$

by (32). Since $\alpha(t)$ and $\gamma(t)$ are uniform limits in $[a, b]$,

$$
\alpha(b)-\alpha(a)+\int_{a}^{b} \alpha(t) d \gamma(t)=b-a .
$$

By the Lebesgue monotone convergence theorem,

$$
\alpha(b)-\alpha(\delta)+\int_{\delta}^{b} \alpha(t) d \gamma(t)=b-\delta .
$$

When $a>\delta$, let $M_{n}(a, t, z)$ and $M(a, t, z)$ be defined by (26) for $m_{n}(t)$ and $m(t)$, respectively. Since $m_{n}(t) \rightarrow m(t)$ uniformly on bounded sets to the right of $a$,

$$
M_{n}(a, b, z) \rightarrow M(a, b, z)
$$

uniformly on bounded subsets of the complex plane; a similar situation occurs in the proof of Theorem XII and the reader should have no difficulty filling in the proof in this case. Since

$$
\alpha_{n}(c)-\alpha_{n}\left(c_{n}\right)+\gamma_{n}(c)-\gamma_{n}\left(c_{n}\right) \rightarrow 0,
$$

the proof of Theorem IX shows that

$$
M_{n}\left(c_{n}, c, z\right) \rightarrow 1
$$

uniformly on every bounded subset of the complex plane. Since (6) holds in the form

$$
\left(A_{n}(c, z), B_{n}(c, z)\right)=\left(A_{n}\left(c_{n}, z\right), B_{n}\left(c_{n}, z\right)\right) M\left(c_{n}, c, z\right)
$$

if $c_{n} \leqq c$, and a similar formula holds when $c \leqq c_{n}$, and since $E_{n}\left(c_{n}, z\right) \rightarrow E(z)$ uniformly on bounded sets, $E_{n}(c, z) \rightarrow E(z)$ uniformly on bounded sets. Let $E(t, z)$ be defined by

$$
E(t, z)=\lim E_{n}(t, z)
$$


for $t>\delta$. By (6), the convergence is uniform on bounded sets, and as we have seen, $E(z)=E(c, z)$. From this definition, it is clear that each such $E(t, z)$ is an entire function satisfying (1) with

$$
B^{\prime}(t, 0)=\lim B_{n}^{\prime}(t, 0)=\lim \alpha_{n}(t)=\alpha(t)>0 .
$$

Formula (6) holds when $\delta<a<b$. We also have

$$
\begin{aligned}
A^{\prime}(t, 0)^{2}-A^{\prime \prime}(t, 0) & =\lim \left[A_{n}^{\prime}(t, 0)^{2}-A_{n}^{\prime \prime}(t, 0)\right] \\
& =2 \lim \int_{0}^{t} \alpha_{n}(s) d \gamma_{n}(s) \\
& =2 \lim \left[t-\alpha_{n}(t)\right] \\
& =2-2 \alpha(t) \\
& =2 \delta+2 \int_{\delta}^{t} \alpha(s) d \gamma(s) .
\end{aligned}
$$

Since each $E_{n}(t, z)$ has genus 0 or 1 by Theorem IV, Lemma 5 applies and in the limit we have the estimate

$$
\begin{aligned}
\log |E(t, z)| \leqq & -x \beta(t)+y \alpha(t)+|z|^{2} \int_{\delta}^{t} \alpha(s) d \gamma(s) \\
& +|z|^{2} \alpha(t)^{2} / 2+|z|^{2} \delta
\end{aligned}
$$

When $\delta \leqq t \leqq c$, the entire functions

$$
E(t, z) \exp [\beta(t) z]
$$

are uniformly bounded on every bounded set. Therefore, there exists a sequence $t_{n} \searrow \delta$ such that

$$
F(z)=\lim E\left(t_{n}, z\right) \exp \left[\beta\left(t_{n}\right) z\right]
$$

exists uniformly on every bounded set. This means that $F(z)$ is an entire function with no zeros for $y>0$, such that

$$
F(0)=1, \quad|F(\bar{z})| \leqq|F(z)| \text { for } y>0, \text { and } \log |F(z)| \leqq \delta|z|^{2}
$$

for all complex $z$. Since

$$
\operatorname{Re}-i F^{\prime}(0)=\lim \alpha\left(t_{n}\right)=\alpha(\delta)=0,
$$

$F(z)$ cannot satisfy (1) and so must satisfy (12). It is an entire function which is real for real $z$, has only real zeros, and

$$
F^{\prime}(0)=0, \quad F^{\prime \prime}(0)=-2 \delta .
$$

By Theorem IV, there exists a family $(E(\delta, t, z)), t \geqq \delta$, of entire functions satisfying (1), such that (6) holds when $\delta<a<b$, and 


$$
E(\delta, t, z) \exp [\beta(t) z] \rightarrow 1,
$$

for all complex $z$ as $t \rightarrow \delta$. By (6) for $E(t, z)$ and $E(\delta, t, z)$,

$$
B(b, z) A(\delta, b, z)-A(b, z) B(\delta, b, z)=B(a, z) A(\delta, a, z)-A(a, z) B(\delta, a, z),
$$

when $\delta<a<b$. Let this entire function be denoted by $L(z)$. It is independent of $a$ or $b$. Since $F(z)$ is real for real $z$,

$$
L(z) \exp \left[\beta\left(t_{n}\right) z\right] \rightarrow 0
$$

for all complex $z$, and therefore $L(z)$ vanishes identically. Now (6) implies that

$$
E(t, z) / E(\delta, t, z)
$$

is independent of $t>\delta$ when $y>0$. On letting $t=t_{n}$ and taking the limit, we find that this ratio is equal to $F(z)$. So,

$$
E(t, z)=F(z) E(\delta, t, z)
$$

for $y>0$, and hence by analyticity, for all complex $z$. Since $E(z)=E(a, z)$ has no real zeros by hypothesis, $F(z)$ has no zeros. Our previous size estimates for this function and the value of its first two derivatives at the origin now show that

$$
F(z)=\exp \left(-\delta z^{2}\right) \text {. }
$$

But $E(z)=E(a, z)$ has genus 0 or 1 by hypothesis and $E(\delta, a, z)$ has genus 0 or 1 by Theorem IV. By Boas [1, p. 27], $\delta=0$ and the theorem follows. In case $E^{*}(z)=E(-z)$, each $E_{n}(z)$ may be chosen so that $E_{n}^{*}(z)=E_{n}(-z)$ by Lemma 1. By Theorem $\mathrm{V}$ in the case already proved, we may choose each $\beta_{n}(t)$ so as to vanish identically. Therefore, $\beta(t)=\lim \beta_{n}(t)$ vanishes identically in this case.

Proof of Theorem VII. For simplicity, we will suppose that $M(z)$ satisfies (4) for $u=0, v=1$. The general case can be obtained by an inner automorphism, as in the proof of Lemma 8. Then, (2) implies that $E(z)=A(z)-i B(z)$ has no zeros for $y \geqq 0$ and that $|E(\bar{z})| \leqq|E(z)|$ for such values of $z$. As in the proof of Lemma $9, E(z)$ satisfies (1) or else is equal to 1 identically. If $E(z)=1$ identically, (2) implies that $\operatorname{Re} D(z) \geqq 1$ for all complex $z$ and hence $D(z)=1$ identically by the argument for $E(z)$. From (2), we have

$$
[\bar{C}(z)-C(z)] /(z-\bar{z}) \geqq 0
$$

for all complex z. By the Poisson representation of a function positive and harmonic in a half plane,

$$
i C(z)-i \bar{C}(z)=i \gamma(\bar{z}-z)
$$

for $y>0$, where $\boldsymbol{\gamma} \geqq 0$ is a constant. By symmetry, the same formula holds for 
all complex z. By the Cauchy-Riemann equations, $C(z)+\gamma z$ is a constant, which is equal to 0 by its value at the origin. Since we have assumed that $M(z)$ satisfies (4) for $u=0, v=1$, we see that $\gamma=0$ and that $M(z)=1$ identically. The theorem certainly holds in this case. Now let us consider the case in which $E(z)$ satisfies (1). Since $E(z)$ has exponential type, it has genus 0 or 1. By Theorem V, $E(z)=E(c, z)$ for some $c \geqq 0$, where $E(t, z)$ is defined as in Theorem IV for some choice of $m(t)$. Actually, the definition of $m(t)$ for $t \geqq b$ is not relevant for the definition of $E(b, z)$. We may suppose with no loss of generality that $b$ is a regular point with respect to $m(t)$. If $a<b$ and $a$ is regular, $\mathfrak{H C}(E(a))$ is contained isometrically in $\mathfrak{H C}(E(b))$ by Theorem IV. By Lemma 12, $[F(z)-F(w)] /(z-w)$ belongs to $\mathfrak{H C}(E(a))$ whenever $F(z)$ belongs to $\mathfrak{H C}(E(a))$, since it belongs to $\mathfrak{H C}(E(b))$ whenever $F(z)$ belongs to $\mathfrak{H C}(E(b))$ by Theorems III and IV of [8]. By these same theorems, there exist entire functions $C(a, z)$ and $D(a, z)$ such that $M(a, z)$ so defined satisfies (2), and (4) with $u=0, v=1$. Since $E(a, 0)=1$, we may suppose, by altering $C(a, z)$ and $D(a, z)$ by constant multiples of $A(a, z)$ and $B(a, z)$, that $M(a, 0)=1$. Then, they are uniquely determined by $A(a, z)$ and $B(a, z)$. Let $M(a, b, z)$ be defined by (26). Since $M(a, b, z)$ satisfies (2) and $M(a, z)$ satisfies (2), and (4) for $u=0$, $v=1$, it is easily verified that

$$
M(b, z)=M(a, z) M(a, b, z)
$$

satifies (2), and (4) for $u=0, v=1$. Certainly, $M(b, 0)=1$ since $M(a, 0)=1$ by construction and $M(a, b, 0)=1$ as a result of (26). By the uniqueness part of Theorem IV of [8], $M(z)=M(b, z)$. Define $M(t, z)$ for $t \geqq a$ by

$$
M(t, z)=M(a, z) M(a, t, z) .
$$

Because of the uniqueness just described, the definition of $M(t, z)$ does not depend on the choice of $a$. If there is a smallest regular point $a>0$, the situation is especially simple. By definition, $\alpha^{\prime}(t), \beta^{\prime}(t), \gamma^{\prime}(t)$ must be equal to constant multiples of a single function in $[0, a]$. Since $m(t)$ was obtained by Theorem IV, $\alpha(a)>\alpha(0)$ and $\alpha^{\prime}(t)$ does not vanish a.e. in this interval. It follows that $\beta^{\prime}(t)$ and $\gamma^{\prime}(t)$ are constant multiples of $\alpha^{\prime}(t)$ in $[0, a]$ and that $m(t)$ satisfies (9). On solving (11), we find that

$$
E(a, z)=[1-\beta(a) z-i \alpha(a) z+\beta(0) z+i \alpha(0) z] \exp [-\beta(0) z],
$$

for all complex $z$. By Theorem IV of [8], $E(a, z)$ satisfies (14) and hence $\beta(0)=0$. Let $M(t, z)$ be defined by (13) for $t>0$. Since (13) implies (11) when $\beta(0)=0$, this does not contradict our previous definition of $A(t, z)$ and $B(t, z)$. By Lemma 10 and the uniqueness part of Theorem IV of [8], this definition of $M(t, z)$ agrees with our previous definition for all $t$. The theorem now follows in the case that there is a smallest regular point $a>0$.

If there is no smallest regular point $a>0$, then $M(t, z)$ has been defined for all $t>0$. Since 


$$
C^{\prime}(a, b, 0)=\gamma(a)-\gamma(b)
$$

whereas

$$
C^{\prime}(b, 0)=C^{\prime}(a, 0)+C^{\prime}(a, b, 0)
$$

by (6), we have

$$
\gamma(a)-\gamma(b)-C^{\prime}(a, b, 0)=-C^{\prime}(a, 0)
$$

and this quantity is nonnegative as a result of (2). Since $\gamma(t)$ is then a nondecreasing function of $t$ which is bounded below, $\gamma(0)=\lim \gamma(t)$ exists and is finite as $t \rightarrow 0$. Now (7) implies that $\beta(0)=\lim \beta(t)$ exists and is finite as $t \rightarrow 0$ and that $m(t)$ satisfies (9). Let $M_{0}(t, z)$ be defined by (13) for $t \geqq 0$. By the uniqueness part of Theorem IV,

$$
E(t, z)=E_{0}(t, z) \exp [-\beta(t) z],
$$

for $t \geqq 0$ and all complex z. By Theorem IV of [8], $E_{0}(t, z)$ satisfies (14). Since $E(t, z)$ also satisfies (14), $\beta(0)=0$. So, $E(t, z)=E_{0}(t, z)$ for $t>0$ and all complex z. As above, the uniqueness part of Theorem IV of [8] implies that $M(t, z)$ $=M_{0}(t, z)$ for $t>0$ and all complex $z$. The theorem follows.

Proof of Theorem VIII. Let $a>0$ be regular with respect to $m(t)$. By Theorem IV, $\mathfrak{F C}(E(b))$ contains $\mathfrak{H C}(E(a))$ isometrically when $b>a$ and $b$ is regular; the same conclusion can be made if $b$ is not regular since the values of $m(t)$ for $t>b$ do not affect the definition of $\mathfrak{F}(E(b))$. Since $E(a, z)$ has no real zeros by Theorem IV, it follows from Theorem VA of [8] that there exists a function $W(a, b, z)$, defined and analytic for $y>0$, such that $|W(a, b, z)| \leqq 1$, and

$$
\frac{y}{\pi} \int \frac{|E(a, t)|^{2}|E(b, t)|^{-2} d t}{(t-x)^{2}+y^{2}}=\operatorname{Re} \frac{E(a, z)+E^{*}(a, z) W(a, b, z)}{E(a, z)-E^{*}(a, z) W(a, b, z)},
$$

for $y>0$. In fact, it was shown in the proof of Theorem VII of [8] that

$$
\frac{1+W(a, b, z)}{1-W(a, b, z)}=\frac{D(a, b, z)+i C(a, b, z)}{A(a, b, z)-i B(a, b, z)} .
$$

Since the functions $W(a, b, z), b \geqq a$, are uniformly bounded for $y>0$, there is a sequence $c_{n} \nearrow \infty$ such that

$$
W(a, z)=\lim W\left(a, c_{n}, z\right)
$$

converges uniformly on every bounded set at a positive distance from the real axis. Therefore, $W(a, z)$ is defined and analytic for $y>0$ and $|W(a, z)| \leqq 1$. By (6) and (33),

$$
W(b, z)=\lim W\left(b, c_{n}, z\right)
$$

is defined and analytic for $y>0$ when $b>a$, and $|W(b, z)| \leqq 1$, and 
(34) $\frac{1+W(a, z)}{1-W(a, z)}=\frac{[D(a, b, z)+i C(a, b, z)]+[D(a, b, z)-i C(a, b, z)] W(b, z)}{[A(a, b, z)-i B(a, b, z)]-[A(a, b, z)+i B(a, b, z)] W(b, z)}$

for $y>0$. By the Poisson representation of a function positive and harmonic in a half plane, there is a nonnegative measure $\mu(b)$ on the Borel sets of the real line and a number $k(b) \geqq 0$ such that

$$
\frac{y}{\pi} \int \frac{|E(b, t)|^{2} d \mu(b, t)}{(t-x)^{2}+y^{2}}+k(b) y=\operatorname{Re} \frac{E(b, z)+E^{*}(b, z) W(b, z)}{E(b, z)-E^{*}(b, z) W(b, z)}
$$

for $y>0$; the factor of $|E(b, t)|^{2}$ in the integrand on the left is permissible since $E(b, z)$ has no real zeros by Theorem IV. We claim that $k(b)=0$ when $b$ is regular. This follows by Theorem VB of [8] unless there are complex numbers $u, v$, not both zero, such that $G(z)$, defined by (5) for $E(z)=E(b, z)$, belongs to $\mathfrak{H C}(E(b))$. In this case, $b$ is the right-hand end point of an interval of singular points by the proof of Theorem IV. For simplicity, we will suppose that $a$ is the largest regular point less than $b$, and that $u$ and $v$ are chosen so that $G(z)$ has norm 1 in $\mathcal{H}(E(b))$. Then, (28) holds for $a \leqq t \leqq b$, and (29) and (30) hold. To show thar $k(b)=0$, we must show that

$$
\frac{[1+W(b, z)] v+[1-W(b, z)] i u}{[1+W(b, z)] i u+[1-W(b, z)] v}
$$

is $o(y)$ when $z=i y$ and $y \rightarrow+\infty$. We apply (34) with $a$ replaced by $b$ and $b$ replaced by $c$, where $c>b$ and $m(t)$ is not constant in $[b, c]$. By the proof of Lemma 10 , it is sufficient to show that]

$$
\frac{[1+W(b, c, z)] v+[1-W(b, c, z)] i u}{[1+W(b, c, z)] i u+[1-W(b, c, z)] v}
$$

is $o(y)$ when $z=i y$ and $y \rightarrow+\infty$. By (33), this is equivalent to showing that $M(b, c, z)$ satisfies (4) for the given $u, v$. This follows from Theorem III since $\mathfrak{F C}(E(b))$ is contained isometrically in $\mathfrak{F C}(E(c))$ by Theorem IV, when $b$ is regular. By the proof of Theorem IX of [8], (34) implies that $\mu(a)=\mu(b)$ when $b$ is regular. Therefore, there is a single nonnegative measure $\mu$ on the Borel sets of the real line such that every $\mathfrak{H C}(E(b))$ is contained isometrically in $L^{2}(\mu)$ when $b$ is regular with respect to $m(t)$.

If there is a largest regular point $b$, the situation is particularly simple. Then, there are complex numbers $u$ and $v$, not both zero, satisfying (3), such that (28) holds when $a$ is replaced by $b$ and $t \geqq b$. A direct computation from (10), using (6), shows that $K(t, z, z) \rightarrow \infty$ for all nonreal $z$ as $t \rightarrow \infty$. It also shows that

$$
W(b, z)=\lim W(b, c, z)
$$

exists as $c \rightarrow \infty$, without going to a subsequence, and that 


$$
W(b, z)=-(u-i v) /(u+i v)
$$

is a constant of absolute value 1. The orthogonal sets of [6] show that $\operatorname{HC}(E(b))$ fills $L^{2}(\mu)$ in this case. For the rest of the proof, we will suppose that the regular points are unbounded. Let $M(z)^{2}=\lim K(t, z, z)$ as $t \rightarrow \infty$. The limit certainly exists since $K(t, z, z)$ is a nondecreasing function of $t$ for each fixed $z$. We must show that the limit is infinite when $z$ is not real. To see this, argue by contradiction, supposing that $M\left(w_{0}\right)$ is finite for some nonreal $w_{0}$. Let $\mathcal{F}_{0}$ be the union of the spaces $\mathcal{H C}(E(b))$, with $b$ regular, and consider it as an inner product space contained isometrically in $L^{2}(\mu)$. Then,

$$
M(z)=\sup |F(z)|,
$$

where $F(z)$ ranges in the elements of $\mathfrak{H}_{0}$ of norm at most 1 . Let $G(z)$ be a fixed element of $\mathcal{H}_{0}$, of norm at most 1 . If $F(z)$ is in $\mathfrak{H}_{0}$ and has norm at most 1 , and if $w$ is not real,

$$
[F(z) G(w)-G(z) F(w)] /(z-w)
$$

belongs to $\mathfrak{H}_{0}$ as a result of (H1) and has norm at most

$$
2[|F(w)|+|G(w)|] /|\bar{w}-w| \text {. }
$$

Therefore,

$$
\left|\frac{F\left(w_{0}\right) G(w)-G\left(w_{0}\right) F(w)}{w_{0}-w}\right| \leqq 2 \frac{|F(w)|+|G(w)|}{|\bar{w}-w|} M\left(w_{0}\right) .
$$

If $w$ is closer to $w_{0}$ than it is to the real axis and if $G(z)$ is chosen so that

$$
\left|G\left(w_{0}\right)\right| /\left|w_{0}-w\right|>2 M\left(w_{0}\right) /|\bar{w}-w|,
$$

the last inequality implies that $M(w)$ is finite, by the arbitrariness of $F(z)$. It also implies that $M(w)$ remains bounded in a neighborhood of $w_{0}$. Since $K(b, \bar{z}, \bar{z})=K(b, z, z)$ for all $b, M(\bar{z})=M(z)$. Since $K(b, x+i y, x+i y)$ is a nondecreasing function of $y \geqq 0$ for each fixed $b$ and $x$ by Lemma 4 and Theorem IV, $M(x+i y)$ is a nondecreasing function of $y \geqq 0$ for each fixed $x$. It follows that $M(z)$ is finite and locally bounded in the complex plane. Let $3 c$ be the Hilbert space of entire functions $F(z)$ in $L^{2}(\mu)$ which are limits of sequences $\left(F_{n}(z)\right)$ in $\mathfrak{F}_{0}$ which converge to $F(z)$ both pointwise and in the metric of $L^{2}(\mu)$. This definition is possible by the properties of $M(z)$, and $\mathcal{H C}$ satisfies (H1), (H2), and (H3). By [7], $\mathfrak{H C}=\mathfrak{H C}(E(\infty))$ for some entire function $E(\infty, z)$ satisfying (1). When $b$ is regular, $\operatorname{FC}(E(b))$ is contained isometrically in $3 C(E(\infty))$, and $E(b, z)$ has no real zeros by Theorem IV. By Theorem III, there is a matrix valued entire function $M(b, \infty, z)$, satisfying $(2)$, such that

$$
(A(\infty, z), B(\infty, z))=(A(b, z), B(b, z)) M(b, \infty, z)
$$

for all complex $z$. Because of Theorem I of [8], the choice of $E(\infty, z)$ can be 
made so that $M(b, \infty, 0)=1$ for at least one value of $b$. By (6) and the uniqueness part of Theorem III,

$$
M(a, \infty, z)=M(a, b, z) M(b, \infty, z)
$$

whenever $b \geqq a$ and $b$ is regular. Since $M(a, b, 0)=1$, we see that $M(b, \infty, 0)=1$ for all regular $b \geqq a$. By taking derivatives at the origin in the last identity, we find that

$$
\begin{aligned}
B^{\prime}(a, \infty, 0) & =\alpha(b)-\alpha(a)+B^{\prime}(b, \infty, 0), \\
-C^{\prime}(a, \infty, 0) & =\gamma(b)-\gamma(a)-C^{\prime}(b, \infty, 0) .
\end{aligned}
$$

But (2) implies that $B^{\prime}(b, \infty, 0) \geqq 0$ and $-C^{\prime}(b, \infty, 0) \geqq 0$, and therefore

$$
\alpha(b)+\gamma(b)-\alpha(a)-\gamma(a) \leqq B^{\prime}(a, \infty, 0)-C^{\prime}(a, \infty, 0) .
$$

This contradiction of (10) shows that $M(z)$ is infinite whenever $z$ is not real.

Now, (34) implies that

$$
W(a, z)=\lim W(a, b, z)
$$

as $b \rightarrow \infty$, since the left-hand side of (33) and the left-hand side of (34) belong to $D(b, z)$ by Lemma 9 , and since the radius of $D(b, z)$ goes to zero as $b \rightarrow \infty$.

It remains to show that the union of the spaces $\mathfrak{H C}(E(b))$, with $b$ regular, is dense in $L^{2}(\mu)$. Let $L$ be an element of $L^{2}(\mu)$ which is orthogonal to every such $\mathfrak{T C}(E(b))$. If $F(z)$ and $G(z)$ are in this union, and if $w$ is not real,

$$
[F(z) G(w)-G(z) F(w)] /(z-w)
$$

is in this union, and therefore

$$
\int \frac{F(t) G(w)-G(t) F(w)}{t-w} \bar{L}(t) d \mu(t)=0 .
$$

Since $w$ is not real,

$$
\begin{aligned}
\left|G(w) \int \frac{F(t) \bar{L}(t) d \mu(t)}{t-w}\right| & =\left|F(w) \int \frac{G(t) \bar{L}(t) d \mu(t)}{t-w}\right| \\
& \leqq 2|F(w)|\|G\||||L||\bar{w}-w|^{-1} .
\end{aligned}
$$

The arbitrariness of $G(z)$ implies that

$$
\int \frac{F(t) \bar{L}(t) d \mu(t)}{t-w}=0
$$

since $M(w)$ is infinite. By the arbitrariness of $w, F(t) \bar{L}(t)$ vanishes a.e. with respect to $\mu$. Since $E(b, z)$ has no real zeros by Theorem IV, for each real $t$ there is an element $F(z)$ of $\mathfrak{T C}(E(b))$ such that $F(t) \neq 0$. Therefore, $L(t)$ vanishes a.e. with respect to $\mu$. This completes the proof that the union of the spaces $\mathfrak{H C}(E(b))$, with $b$ regular, is dense in $L^{2}(\mu)$. 
Proof of Theorem IX. By Theorem VII, $M(z)=M(a, z)$ for some $a \geqq 0$, where $M(t, z)$ is defined as in Theorem VI for some choice of $m(t)$. Let it be chosen so that $m(0)=0$. Let

$$
M(t, z)=\sum M_{n}(t) z^{n}
$$

be the power series expansion. Then, $M_{0}(t)=1$,

$$
M_{1}(t)=\left(\begin{array}{ll}
-\beta(t) & \alpha(t) \\
-\gamma(t) & \beta(t)
\end{array}\right),
$$

each $M_{n}(t)$ is a continuous function of $t \geqq 0$, and

$$
M_{n+1}(t) I=\int_{0}^{t} M_{n}(s) m^{\prime}(s) d s .
$$

By (7),

$$
\left\|m^{\prime}(t)\right\|^{2} \leqq \alpha^{\prime}(t)^{2}+2 \beta^{\prime}(t)^{2}+\gamma^{\prime}(t)^{2} \leqq\left[\alpha^{\prime}(t)+\gamma^{\prime}(t)\right]^{2}
$$

a.e., for $t \geqq 0$. Therefore,

$$
\left\|M_{n+1}(t)\right\| \leqq \int_{0}^{t}\left\|M_{n}(s)\right\|\left[\alpha^{\prime}(s)+\gamma^{\prime}(s)\right] d s .
$$

Since $\left\|M_{0}(t)\right\|=1$, we find inductively that

$$
n !\left\|M_{n}(t)\right\| \leqq[\alpha(t)+\gamma(t)]^{n} .
$$

The theorem follows.

Proof of Theorem X. As we have seen, for each $t \geqq 0, A(t, z), \cdots, D(t, z)$ are entire functions of exponential type which are real for real $z$ and satisfy (14). By Boas [1, p. 116], when $\theta \neq 0, \pi$,

$$
\lim r^{-1} \log \left|F\left(r e^{i \theta}\right)\right|=\tau|\sin \theta|
$$

holds for each of these entire functions, with $\tau$ equal to the type of $F(z)$. It follows from (2) that each of the functions

$$
\begin{gathered}
{[D(t, z)+i C(t, z)] /[A(t, z)-i B(t, z)],} \\
i A(t, z) / B(t, z), \quad i C(t, z) / D(t, z)
\end{gathered}
$$

and its reciprocal is defined and analytic for $y>0$ and has a nonnegative real part. By the Poisson representation, each of these functions and its reciprocal is $O(y)$ on the upper half of the imaginary axis, as $y \rightarrow \infty$. Therefore, each of the entire functions $A(t, z), \cdots, D(t, z)$ has the same type $\tau(0, t)$. We will prove the theorem by showing that $\tau(0, t)=\tau(t)$. Let

$$
\begin{aligned}
& F(t, z)=A(t, z) \exp [i \tau(t) z], \\
& G(t, z)=B(t, z) \exp [i \tau(t) z] .
\end{aligned}
$$


For each fixed $t, F(t, z)$ and $G(t, z)$ are entire functions of $z$. For each fixed $z$, $F(t, z)$ and $G(t, z)$ are absolutely continuous functions of $t$ such that

$$
\begin{aligned}
& {[G(t, z) \bar{F}(t, z)-F(t, z) \bar{G}(t, z)]^{\prime} /(z-\bar{z}) } \\
&= F(t, z) \bar{F}(t, z) \alpha^{\prime}(t)+G(t, z) \bar{F}(t, z)\left[\beta^{\prime}(t)+i \tau^{\prime}(t)\right] \\
&+F(t, z) \bar{G}(t, z)\left[\beta^{\prime}(t)-i \tau^{\prime}(t)\right]+G(t, z) \bar{G}(t, z) \gamma^{\prime}(t) \\
& \geqq 0,
\end{aligned}
$$

a.e. as a result of $(7)$ and the definition of $\tau(t)$. Therefore,

$$
[G(t, z) \bar{F}(t, z)-F(t, z) \bar{G}(t, z)] /(z-\bar{z}) \geqq 0
$$

is a nondecreasing function of $t \geqq 0$. It follows that

$$
\tau(0, t)-\tau(t) \geqq 0
$$

is a nondecreasing function of $t \geqq 0$. When $a \leqq b$, let $M(a, b, z)$ be defined by (26). The argument just gone through shows that each of the entire functions $A(a, b, z), \cdots, D(a, b, z)$ has the same type $\tau(a, b)$ and that

$$
\tau(a, b) \geqq \tau(b)-\tau(a) .
$$

Because of (6), we also have

$$
\tau(0, b) \leqq \tau(0, a)+\tau(a, b) .
$$

Let

$$
P=\left(\begin{array}{ll}
p & q \\
q & r
\end{array}\right)
$$

where $p, q, r$ are real numbers such that $p r-q^{2}=1$. If $M_{n}(t)$ is defined as in the proof of Theorem IX,

$$
M_{n+1}(t) P=-\int_{0}^{t} M_{n}(s) P P^{-1} m^{\prime}(s) I P d s
$$

and so

$$
\left.\left\|M_{n+1}(t) P\right\| \leqq \int_{0}^{t}\left\|M_{n}(s) P\right\| \| P^{-1} m^{\prime} s\right) I P \| d s .
$$

Since $\left\|M_{0}(t) P\right\|=\|P\|$, we see inductively that

$$
n !\left\|M_{n}(t) P\right\| \leqq\|P\|\left(\int_{0}^{t}\left\|P^{-1} m^{\prime}(s) I P\right\| d s\right)^{n}
$$

and therefore

$$
\log \|M(t, z) P\| \leqq \log \|P\|+|z| \int_{0}^{t}\left\|P^{-1} m^{\prime}(s) I P\right\| d s .
$$


Therefore,

$$
\tau(0, t) \leqq \int_{0}^{t}\left\|P^{-1} m^{\prime}(s) I P\right\| d s .
$$

A similar argument will show that

$$
\tau(a, b) \leqq \int_{a}^{b}\left\|P^{-1} m^{\prime}(t) I P\right\| d t
$$

when $0 \leqq a \leqq b$. Since

$$
0 \leqq \tau(0, b)-\tau(0, a) \leqq \tau(a, b),
$$

the last inequality implies that $\tau(0, t)$ is an absolutely continuous function of $t$ and

$$
\tau^{\prime}(0, t) \leqq\left\|P^{-1} m^{\prime}(t) I P\right\|
$$

a.e. Since the $2 \times 2$ matrices with real entries are a separable metric space,

$$
\tau^{\prime}(0, t) \leqq \inf \left\|P^{-1} m^{\prime}(t) I P\right\|
$$

where $p, q, r$ are allowed real values such that $p r-q^{2}=1$. If for some real t. $m^{\prime}(t)$ exists and $\tau^{\prime}(t)>0$, choose $p, q, r$ so that

$$
\begin{aligned}
& \alpha^{\prime}(t)=\left(p^{2}+q^{2}\right) \tau^{\prime}(t), \\
& \beta^{\prime}(t)=(p q+q r) \tau^{\prime}(t), \\
& \gamma^{\prime}(t)=\left(q^{2}+r^{2}\right) \tau^{\prime}(t) .
\end{aligned}
$$

Then,

$$
\left\|P^{-1} m^{\prime}(t) I P\right\|=\left\|I \tau^{\prime}(t)\right\|=\tau^{\prime}(t),
$$

for this choice of $P$. When $\tau^{\prime}(t)=0$,

$$
\inf \left\|P^{-1} m^{\prime}(t) I P\right\|=0,
$$

by continuity. We have shown that $\tau^{\prime}(0, t) \leqq \tau^{\prime}(t)$ a.e. and so $\tau(0, t) \leqq \tau(t)$ for $t \geqq 0$, since both functions vanish at the origin. We have already obtained the reverse inequality. So, $\tau(0, t)=\tau(t)$ and the theorem follows.

Proof of Theorem XI. For $y>0$

$$
f(z)=\lim i C(b, z) / A(b, z)
$$

exists as $b \rightarrow \infty$ by Lemma 9 since the fraction on the right belongs to $D(b, z)$ and the radius of $D(b, z)$ goes to zero by Theorem VIII. Since the convergence is bounded on bounded sets at a positive distance from the real axis, $f(z)$ is defined and analytic for $y>0$. Since $f(z)$ belongs to $D(b, z), \operatorname{Re} f(z) \geqq 0$ for $y>0$. Since $\alpha(t)>\alpha(0)$ by hypothesis, each $M(b, z)$ satisfies (4) for $u=0, v=1$ by Lemma 10. For each fixed $b$, 


$$
[D(b, i y)+i C(b, i y)] /[A(b, i y)-i B(b, i y)]
$$

belongs to $D(b, i y)$ and is $o(y)$ as $y \rightarrow+\infty$. Since the radius of $D(b, i y)$ remains bounded as $y \rightarrow+\infty$ for each fixed $b$, by Lemma 4, we have $f(i y)=o(y)$ as $y \rightarrow+\infty$. By the Poisson representation of a function positive and harmonic in a half plane, (17) does define a nonnegative measure $\mu$. As in the proof of Theorem VIII, let

$$
W(a, z)=\lim W(a, b, z)
$$

as $b \rightarrow \infty$, where $W(a, b, z)$ is defined by (33). Then, for each $a>0$,

$$
\begin{aligned}
\frac{y}{\pi} \int \frac{d \mu(t)}{(t-x)^{2}+y^{2}} & \\
= & \operatorname{Re} \frac{[D(a, z)+i C(a, z)]+[D(a, z)-i C(a, z)] W(a, z)}{[A(a, z)-i B(a, z)]-[A(a, z)-i B(a, z)] W(a, z)},
\end{aligned}
$$

for $y>0$, because of (6) and (17), and because the radius of $D(b, z)$ goes to zero as $b \rightarrow \infty$. Let the measure $\mu(b)$ be defined by (35) when $b$ is regular with respect to $m(t)$; as in the proof of Theorem VIII, $k(b)=0$. By the proof of Theorem VI of $[8], \mu(b)=\mu$. The theorem now follows from Theorem VIII.

Proof of Theorem XII, when $\mu$ is supported at a finite number $r$ of points. Since $\mu$ does not vanish identically by hypothesis, $r>0$. Consider the polynomials of degree at most $r-1$ in the norm of $L^{2}(\mu)$. They form a Hilbert space of entire functions which satisfies (H1), (H2), and (H3) and which contains a nonzero element, the constant function 1. By [7], this Hilbert space is equal isometrically to some $\mathfrak{H}(E)$, for some entire function $E(z)$ satisfying (1). By Theorem I of [8], we may choose $E(z)$ so that $E(0)=1$. Since $K(w, z)$ belongs to $\mathfrak{H}(E)$ for every complex number $w$, it has degree at most $r-1$, and has degree $r-1$ for some values of $w$; so, $E(z)$ is a polynomial of degree $r$. As in the proof of Theorem $\mathrm{V}$ when $E(z)$ is polynomial, there are polynomials $C(z)$ and $D(z)$ of degree at most $r$ such that $M(z)$ so defined satisfies (2), and (4) for $u=0, v=1$, and $M(0)=1$. Furthermore, $M(z)=M(a, z)$ for some $a>0$, where $M(t, z)$ is defined by (13) for some choice of $m(t)$ satisfying (7), (8), and (9). Since the values of $m(t)$ for $t \geqq a$ do not affect the definition of $M(a, z)$, $m(t)$ may be chosen so that $a$ is a regular point with respect to $m(t)$, and there are no regular points greater than $a$, and $m(t)$ satisfies (10). To do this, let $u$ and $v$ be complex numbers, not both zero, which satisfy (3), and define $m(t)$ and $M(a, t, z)$ so that (28) holds for $t \geqq a$. The choice of $u$ and $v$ must be such that $G(z)$, defined by $(5)$ for $E(z)=E(a, z)$, does not belong to $\mathfrak{H}(E(a))$. For the right choice of $u$ and $v$, note by Theorem $\mathrm{V}$ of [8] that (35) must hold with $b$ replaced by $a$ and $k(a)=0$, since $\mathfrak{F C}(E(a))$ is contained isometrically in $L^{2}(\mu)$ and $E(a, z)$ has no real zeros. Since $\mathfrak{T}(E)$ fills $L^{2}(\mu)$ by construction, $W(a, z)$ is a constant of absolute value 1 because of the orthogonal sets of [6]. Let $u$ and $v$ be chosen so that 


$$
W(a, z)=-(u-i v) /(u+i v) .
$$

As in the proof of Theorem XI, (37) holds. It is easily verified that $m(t)$ so constructed has the required properties.

Proof of Theorem XII. Let $\left(\mu_{n}\right)$ be a sequence of nonnegative measures on the Borel sets of the real line, satisfying the hypotheses of the theorem and for which the conclusion has already been obtained, such that

$$
\frac{y}{\pi} \int \frac{d \mu(t)}{(t-x)^{2}+y^{2}}=\lim \frac{y}{\pi} \int \frac{d \mu_{n}(t)}{(t-x)^{2}+y^{2}}
$$

for $y>0$. This approximation is possible because the theorem has been established for measures supported on finite sets. For each $n$, let $m_{n}(t)$ and $M_{n}(t, z)$ be constructed for $\mu_{n}$ as the theorem states. By altering $m_{n}(t)$ by a constant, we may suppose it chosen so that $m_{n}(0)=0$. By a reparametrization, we may suppose that

$$
\alpha_{n}(t)+\gamma_{n}(t)=t
$$

for every $n$. Then, (7) implies that $\left(\alpha_{n}\right),\left(\beta_{n}\right),\left(\gamma_{n}\right)$ are uniformly bounded, equicontinuous sequences of functions for $t$ in any bounded set. We may suppose by passing to a subsequence that $m(t)=\lim m_{n}(t)$ converges uniformly on bounded sets. Therefore, $m(t)$ satisfies (7), (8), (9) with $m(0)=0$, and (10) with

$$
\alpha(t)+\gamma(t)=t
$$

Let $M(t, z)$ be defined by (13) for this choice of $m(t)$. Since $m_{n}(t) \rightarrow m(t)$ uniformly on bounded sets, $M_{n}(t, z) \rightarrow M(t, z)$ in the formal sense. That is, when these analytic functions are expanded in Taylor series about the origin and $t$ is held fixed, the $r$ th coefficient of the series on the left converges to the $r$ th coefficient of the series on the right for every $r$. This follows inductively from (36). As a result of Theorem IX, the convergence is now uniform on bounded subsets of the complex plane, for each fixed $t$. But for each $a>0$, (37) holds for $y>0$, with $\mu=\mu_{n}, M(a, z)=M_{n}(a, z), W(a, z)=W_{n}(a, z)$. Because of (38),

$$
W(a, z)=\lim W_{n}(a, z)
$$

exists for $y>0$ and (37) holds. By the Lebesgue dominated convergence theorem, the right-hand side of (37) is $o(y)$ when $z=i y$ and $y \rightarrow+\infty$. By Lemma 9, the right-hand side of (37) belongs to $D(a, z)$; the same is true for the righthand side of (33) when $a$ is replaced by 0 and $b$ by $a$. The radius of $D(a, i y)$ remains bounded as $y \rightarrow+\infty$ with $a$ held fixed, by Lemma 4 . It follows that $M(a, z)$ satisfies (4) with $u=0, v=1$. By Lemma 10, $\alpha(t)>\alpha(0)$ for $t>0$. Formula (17) now follows from Lemma 9 since the radius of $D(a, z)$ goes to zero as $a \rightarrow \infty$, by Theorem VIII. 


\section{REFERENCES}

1. R. P. Boas, Jr., Entire functions, New York, Academic Press, 1954.

2. L. de Branges, Local operators on Fourier transforms, Duke Math. J. vol. 25 (1958) pp. 143-154.

3. - The a-local operator problem, Canad. J. Math. vol. 11 (1959) pp. 583-592.

4. - The Stone-Weierstrass theorem, Proc. Amer. Math. Soc. vol. 10 (1959) pp. 822824.

5. - The Bernstein problem, Proc. Amer. Math. Soc. vol. 10 (1959) pp. 825-832.

6. - Some mean squares of entire functions, Proc. Amer. Math. Soc. vol. 10 (1959) pp. 833-839.

7. - Some Hilbert spaces of entire functions, Proc. Amer. Math. Soc. vol. 10 (1959) pp. 840-846.

8. - Some Hilbert spaces of entire functions, Trans. Amer. Math. Soc. vol. 96 (1960) pp. 259-295.

The Institute for Advanced Study,

Princeton, New Jersey 